\title{
An asymptotic method based on a Hopf-Cole transformation for a kinetic BGK equation in the hyperbolic limit
}

\author{
Songting Luo ${ }^{\mathrm{a}, *}$, Nicholas Payne ${ }^{\mathrm{a}}$ \\ ${ }^{a}$ Department of Mathematics, Iowa State University, Ames, IA 50011.
}

\begin{abstract}
We present an effective asymptotic method for approximating the density of particles for kinetic equations with a Bhatnagar-Gross-Krook (BGK) relaxation operator in the large scale hyperbolic limit. The density of particles is transformed via a Hopf-Cole transformation, where the phase function is expanded as a power series with respect to the Knudsen number. The expansion terms can be determined by solving a sequence of equations. In particular, it has been proved in 3 that the leading order term is the viscosity solution of an effective Hamilton-Jacobi equation, and we show that the higher order terms can be formally determined by solving a sequence of transport equations. Both the effective Hamilton-Jacobi equation and the transport equations are independent of the Knudsen number, and are formulated in the physical space, where the effective Hamiltonian is obtained as the solution of a nonlinear equation that is given as an integral in the velocity variable, and the coefficients of the transport equations are given as integrals in the velocity variable. With appropriate Gauss quadrature rules for evaluating these integrals effectively, the effective Hamilton-Jacobi equation and the transport equations can be solved efficiently to obtain the expansion terms for approximating the density function. In this work, the zeroth, first and second order terms in the expansion are used to obtain second order accuracy with respect to the Knudsen number. The proposed method balances efficiency and accuracy, and has the potential to deal with kinetic equations with more general BGK models. Numerical experiments verify the effectiveness of the proposed method.
\end{abstract}

Keywords: kinetic equation, BGK operator, Hopf-Cole transformation, asymptotic method 2010 MSC: 65M06, 35Q20, 35Q83, 35F21

\footnotetext{
This work is partially supported by NSF DMS 1418908 .

* Corresponding author

Email addresses: luos@iastate.edu (Songting Luo), napayne@iastate.edu (Nicholas Payne)
} 


\section{Introduction}

We consider the following kinetic equation with a Bhatnagar-Gross-Krook (BGK) relaxation operator in the large scale hyperbolic limit [2,

$$
\partial_{t} f^{\epsilon}(t, \mathbf{x}, \mathbf{v})+\mathbf{v} \cdot \nabla_{\mathbf{x}} f^{\epsilon}(t, \mathbf{x}, \mathbf{v})=\frac{1}{\epsilon}\left(M(\mathbf{v}) \rho^{\epsilon}(t, \mathbf{x})-f^{\epsilon}(t, \mathbf{x}, \mathbf{v})\right),(t, \mathbf{x}, \mathbf{v}) \in \mathbb{R}_{+} \times \mathbb{R}^{n} \times \mathbb{V}
$$

where $f^{\epsilon}(t, \mathbf{x}, \mathbf{v})$ denotes the density of particles moving with velocity $\mathbf{v} \in \mathbb{V}$ at time $t \in \mathbb{R}_{+}$and position $\mathbf{x} \in \mathbb{R}^{n}, \mathbb{V} \subset \mathbb{R}^{n}$ is bounded and symmetric, $\epsilon>0$ is proportional to the Knudsen number, $\rho^{\epsilon}(t, \mathbf{x})$ denotes the macroscopic density of particles, i.e.,

$$
\rho^{\epsilon}(t, \mathbf{x})=\int_{\mathbb{V}} f^{\epsilon}(t, \mathbf{x}, \mathbf{v}) d \mathbf{v},(t, \mathbf{x}) \in \mathbb{R}_{+} \times \mathbb{R}^{n}
$$

and $M$ is a fixed Maxwellian density which is symmetric and satisfies the following moment identities,

$$
\int_{\mathbb{V}} M(\mathbf{v}) d \mathbf{v}=1, \int_{\mathbb{V}} \mathbf{v} M(\mathbf{v}) d \mathbf{v}=\mathbf{0}, \int_{\mathbb{V}} \mathbf{v}^{2} M(\mathbf{v}) d \mathbf{v}=\theta^{2}
$$

The BGK model is a relaxation model associated with the Boltzmann equation for the kinetic description of gases. It provides a much simpler form of the collision term and retains the principal effects of particle collisions, which enables more tractable solution methods. The BGK model is known to be accurate in describing systems close to equilibrium [6. The importance of the BGK model in applications has motivated the development of many numerical methods tailored to particular structures of the equations; refer to the survey paper 8 and references therein. The main difficulties for solving such equations are due to the highdimensional structure of the density of particles and the presence of multiple scales. In a three-dimensional (3-D) simulation, the problem is 3 (space) +3 (velocity) +1 (time) dimensional. For small values of $\epsilon$, the problem is stiff and standard time discretization methods are forced to operate on a very small time scale. Therefore, the problem is highly challenging, and an effective numerical method must balance accuracy and efficiency.

In this work, we use equation (1) to propose and explain an asymptotic approach to approximate the density of particles, aiming to balance efficiency and accuracy. The method is based on the Hopf-Cole (HC) transformation [11]. As $\epsilon \rightarrow 0$, the density of particles relaxes rapidly towards the Maxwellian distribution, which motivates the following HC transformation of the density,

$$
f^{\epsilon}(t, \mathbf{x}, \mathbf{v})=M(\mathbf{v}) \exp \left(-\frac{\psi^{\epsilon}(t, \mathbf{x}, \mathbf{v})}{\epsilon}\right)
$$


where $\psi^{\epsilon}$ is the phase function. It has been proved rigorously in 3$]$ that $\psi^{\epsilon}$ satisfies the following properties.

Proposition 1.1 ([3]). Let $\mathbb{V} \subset \mathbb{R}^{n}$ be a bounded subset. Assume $M \in L^{1}(\mathbb{V})$ is nonnegative and symmetric, and satisfies the moment identities $(2), \psi^{\epsilon}(0, \boldsymbol{x}, \boldsymbol{v})=\psi_{0}(\boldsymbol{x}) \geq 0$, and $\psi_{0} \in W^{1, \infty}\left(\mathbb{R}^{n}\right)$, then $\psi^{\epsilon} \in W^{1, \infty}\left(\mathbb{R}_{+} \times\right.$ $\mathbb{R}^{n} \times \mathbb{V}$ ) and satisfies the following uniform estimates,

$$
\begin{aligned}
& 0 \leq \psi^{\epsilon}(t, \cdot, \cdot) \leq\left\|\psi_{0}\right\|_{\infty}, \\
& \left\|\nabla_{x} \psi^{\epsilon}(t, \cdot, \cdot)\right\|_{\infty} \leq\left\|\nabla_{x} \psi_{0}\right\|_{\infty}, \\
& \left\|\nabla_{\boldsymbol{v}} \psi^{\epsilon}(t, \cdot, \cdot)\right\|_{\infty} \leq t\left\|\nabla_{x} \psi_{0}\right\|_{\infty}, \\
& \left\|\partial_{t} \psi^{\epsilon}(t, \cdot, \cdot)\right\|_{\infty} \leq V_{\max }\left\|\nabla_{x} \psi_{0}\right\|_{\infty},
\end{aligned}
$$

where $V_{\max }$ is the maximum modulus of velocity.

We shall expand the phase function $\psi^{\epsilon}$ as a power series,

$$
\psi^{\epsilon}(t, \mathbf{x}, \mathbf{v})=\psi^{0}(t, \mathbf{x}, \mathbf{v})+\epsilon \psi^{1}(t, \mathbf{x}, \mathbf{v})+\epsilon^{2} \psi^{2}(t, \mathbf{x}, \mathbf{v})+\cdots+\epsilon^{k} \psi^{k}(t, \mathbf{x}, \mathbf{v})+\cdots
$$

as $\epsilon \rightarrow 0$, where $\left\{\psi^{k}\right\}_{k=0}^{\infty}$ are the expansion terms. By substituting the expansion (5) into equation (1) and collecting terms of same orders in $\epsilon$, we shall show later that one can formally derive governing equations for the sequence $\left\{\psi^{k}\right\}_{k=0}^{\infty}$. In particular, it has been shown in [3, 4] that the leading order term $\psi^{0}$ can be obtained as the viscosity solution of an effective Hamilton-Jacobi (HJ) equation. We show formally that higher order terms $\left\{\psi^{k}\right\}_{k=1}^{\infty}$ can be determined with solutions of a sequence of transport equations. The effective HJ equation and the transport equations are formulated in the physical space with coefficients given as integrals in the velocity variable, and they are independent of $\epsilon$.

Since the governing equations for $\left\{\psi^{k}\right\}_{k=0}^{\infty}$ are formulated in the physical space, they do not depend on $\epsilon$, and their coefficients can be evaluated with appropriate Gauss quadrature rules, we can solve them efficiently and use the solutions to approximate the phase function and the density function with formulae (5) and (3) respectively. The overall complexity is expected to be significantly reduced compared to that of dealing with the BGK model directly in the phase space. In this work, we will use the zeroth, first and second order terms, $\left\{\psi^{0}, \psi^{1}, \psi^{2}\right\}$, to approximate $\psi^{\epsilon}$ and $f^{\epsilon}$ by

$$
\psi^{\epsilon} \approx \psi^{0}+\epsilon \psi^{1}+\epsilon^{2} \psi^{2}, \text { and } f^{\epsilon} \approx M(\mathbf{v}) \exp \left(-\frac{\psi^{0}}{\epsilon}-\psi^{1}-\epsilon \psi^{2}\right)
$$

This approximation of $f^{\epsilon}$ is formally $O\left(\epsilon^{2}\right)$ accurate as $\epsilon \rightarrow 0$. And efficient finite difference schemes will be designed to numerically solve the effective HJ equation and the transport equations for $\left\{\psi^{0}, \psi^{1}, \psi^{2}\right\}$. 
The proposed approach is related to the Chapman-Enskog theory [5], where $f^{\epsilon}$ is expanded as

$$
f^{\epsilon}(t, \mathbf{x}, \mathbf{v})=f^{0}(t, \mathbf{x}, \mathbf{v})+\epsilon f^{1}(t, \mathbf{x}, \mathbf{v})+\epsilon^{2} f^{2}(t, \mathbf{x}, \mathbf{v})+\cdots=\sum_{k=0}^{\infty} \epsilon^{k} f^{k}(t, \mathbf{x}, \mathbf{v})
$$

with $\left\{f^{k}\right\}_{k=0}^{\infty}$ determined through a sequence of equations in the phase space, and higher order terms depend on lower order terms. From the HC transformations (3) and the power series expansion (5), we have

$$
\begin{aligned}
f^{\epsilon}(t, \mathbf{x}, \mathbf{v}) & =M(\mathbf{v}) \exp \left(-\frac{\psi^{0}(t, \mathbf{x}, \mathbf{v})}{\epsilon}-\psi^{1}(t, \mathbf{x}, \mathbf{v})\right) \exp \left(-\sum_{k=1}^{\infty} \epsilon^{k} \psi^{k+1}(t, \mathbf{x}, \mathbf{v})\right) \\
& =M(\mathbf{v}) \exp \left(-\frac{\psi^{0}}{\epsilon}-\psi^{1}\right)\left(1+\epsilon\left(-\psi^{2}\right)+\epsilon^{2}\left(\left(\psi^{2}\right)^{2}-\psi^{3}\right)+\epsilon^{3}\left(-\left(\psi^{2}\right)^{2}+\psi^{2} \psi^{3}-\psi^{4}\right)+\cdots\right),
\end{aligned}
$$

which can be rewritten as the expansion (7). The main difference between the proposed method and the Chapman-Enskog expansion is that $\left\{\psi^{k}\right\}_{k=0}^{\infty}$ can be determined with solutions of a sequence of equations that are formulated in the physical space, which is analogous to the semiclassical approximation and Wentzel-Kramers-Brillouin (WKB) approximation in the computational high frequency wave propagation 14, 13, 9, 18, 19. Therefore, the equations are numerically easier to solve with a much lower complexity.

The rest of the paper is organized as follows. In Section 2 we present the effective HJ equation and the transport equations for determining the expansion terms. In Section 3 we present numerical schemes for solving the equations and approximating the density function. In Section 4 , numerical experiments are performed to demonstrate the proposed method. Concluding remarks along with discussion of future projects are given at the end.

\section{Governing Equations}

In this section, we recall the results from [3, 4] on the effective Hamilton-Jacobi equation for $\psi^{0}$, and derive formally the governing equations for $\psi^{1}$ and $\psi^{2}$.

\subsection{Effective Hamilton-Jacobi Equation for $\psi^{0}$}

It has been proved that $\psi^{\epsilon}(t, \mathbf{x}, \mathbf{v}) \rightarrow \psi^{0}(t, \mathbf{x})$ (locally) uniformly as $\epsilon \rightarrow 0$. The limit function $\psi^{0}$ is independent of $\mathbf{v}$ and is the viscosity solution of an effective HJ equation [3, 4]. As in 4, we denote $\operatorname{Conv}(\mathbb{V})$ as the convex hull of $\mathbb{V}$, and define

$$
\mu(\mathbf{p}) \equiv \max _{\mathbf{v} \in \operatorname{Conv}(\mathbb{V})}\{\mathbf{v} \cdot \mathbf{p}\}, \operatorname{Sing}(M) \equiv\left\{\mathbf{p} \in \mathbb{R}^{n}: \int_{\mathbb{V}} \frac{M(\mathbf{v})}{\mu(\mathbf{p})-\mathbf{v} \cdot \mathbf{p}} d \mathbf{v} \leq 1\right\}
$$




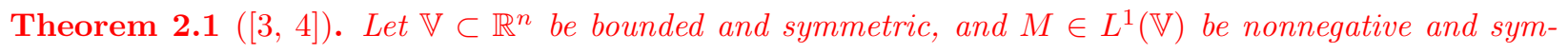
metric, and $M$ satisfies the moment identities in 22. Then $\psi^{\epsilon}$ converges (locally) uniformly towards $\psi^{0}$, where $\psi^{0}$ is the viscosity solution of the following effective HJ equation,

$$
\partial_{t} \psi^{0}(t, \boldsymbol{x})+H\left(\nabla_{x} \psi^{0}(t, \boldsymbol{x})\right)=0,
$$

with the effective Hamiltonian $H(\boldsymbol{p})$ for $\boldsymbol{p}=\nabla_{x} \psi^{0}(t, \boldsymbol{x})$ defined as follows,

- if $\boldsymbol{p} \in \operatorname{Sing}(M), H(\boldsymbol{p})=\mu(\boldsymbol{p})-1$;

- if $\boldsymbol{p} \notin \operatorname{Sing}(M), H(\boldsymbol{p})$ is uniquely given as the solution of the equation

$$
\int_{\mathbb{V}} \frac{M(\boldsymbol{v})}{1+H(\boldsymbol{p})-\boldsymbol{v} \cdot \boldsymbol{p}} d \boldsymbol{v}=1
$$

such that $1+H(\boldsymbol{p})-\boldsymbol{v} \cdot \boldsymbol{p}>0$ for all $\boldsymbol{v} \in \mathbb{V}$. That is,

$$
\int_{\mathbb{V}} \frac{M(\boldsymbol{v})}{1-\partial_{t} \psi^{0}(t, \boldsymbol{x})-\boldsymbol{v} \cdot \nabla_{\boldsymbol{x}} \psi^{0}(t, \boldsymbol{x})} d \boldsymbol{v}=1,(t, \boldsymbol{x}) \in \mathbb{R}_{+} \times \mathbb{R}^{n}
$$

In this work, we focus on cases when $\operatorname{Sing}(M)=\emptyset$ as in $\underline{3}$. The cases with $\operatorname{Sing}(M) \neq \emptyset$ will be considered in a future work.

The limit function $\psi^{0}$ is the leading order term in the power series expansion (5).

By differentiating equation (9) with respect to $\mathbf{p}$, we have

$$
\int_{\mathbb{V}} \frac{M(\mathbf{v})\left(\nabla_{\mathbf{p}} H(\mathbf{p})-\mathbf{v}\right)}{(1+H(\mathbf{p})-\mathbf{v} \cdot \mathbf{p})^{2}} d \mathbf{v}=0
$$

which implies

$$
\nabla_{\mathbf{p}} H(\mathbf{p}) \int_{\mathbb{V}} \frac{M(\mathbf{v})}{(1+H(\mathbf{p})-\mathbf{v} \cdot \mathbf{p})^{2}} d \mathbf{v}=\int_{\mathbb{V}} \frac{\mathbf{v} M(\mathbf{v})}{(1+H(\mathbf{p})-\mathbf{v} \cdot \mathbf{p})^{2}} d \mathbf{v},
$$

and $\left|\nabla_{\mathbf{p}} H(\mathbf{p})\right| \leq V_{\max }[3$. By differentiating equation (9) with respect to $\mathbf{p}$ twice, we have

$$
\int_{\mathbb{V}} \frac{\left.M(\mathbf{v})\left[D_{\mathbf{p}}^{2} H(\mathbf{p})(1+H(\mathbf{p})-\mathbf{v} \cdot \mathbf{p})^{2}\right)-2(1+H(\mathbf{p})-\mathbf{v} \cdot \mathbf{p})\left(\nabla_{\mathbf{p}} H(\mathbf{p})-\mathbf{v}\right) \otimes\left(\nabla_{\mathbf{p}} H(\mathbf{p})-\mathbf{v}\right)\right]}{(1+H(\mathbf{p})-\mathbf{v} \cdot \mathbf{p})^{4}} d \mathbf{v}=0,
$$

which implies

$$
D_{\mathbf{p}}^{2} H(\mathbf{p}) \int_{\mathbb{V}} \frac{M(\mathbf{v})}{(1+H(\mathbf{p})-\mathbf{v} \cdot \mathbf{p})^{2}} d \mathbf{v}=2 \int_{\mathbb{V}} \frac{M(\mathbf{v})\left[\left(\nabla_{\mathbf{p}} H(\mathbf{p})-\mathbf{v}\right) \otimes\left(\nabla_{\mathbf{p}} H(\mathbf{p})-\mathbf{v}\right)\right]}{(1+H(\mathbf{p})-\mathbf{v} \cdot \mathbf{p})^{3}} d \mathbf{v} .
$$


Equation 12 implies that $H(\mathbf{p})$ is convex 3 .

45 2.2. Transport Equations for $\psi^{1}$ and $\psi^{2}$

We continue to derive the governing equations for $\psi^{1}$ and $\psi^{2}$.

Proposition 2.1. Under the same assumptions in Theorem 2.1 and assume $\operatorname{Sing}(M)=\emptyset, \psi^{1}(t, \boldsymbol{x}, \boldsymbol{v})$ is given by

$$
\psi^{1}(t, \boldsymbol{x}, \boldsymbol{v})=\log G(t, \boldsymbol{x}, \boldsymbol{v})-\lambda(t, \boldsymbol{x})
$$

where

$$
G(t, \boldsymbol{x}, \boldsymbol{v}) \equiv 1-\partial_{t} \psi^{0}(t, \boldsymbol{x})-\boldsymbol{v} \cdot \nabla_{x} \psi^{0}(t, \boldsymbol{x}),
$$

and $\lambda(t, x)$ satisfies the following transport equation,

$$
\partial_{t} \lambda(t, \boldsymbol{x})+\frac{\boldsymbol{B}(t, \boldsymbol{x})}{a(t, \boldsymbol{x})} \cdot \nabla_{\boldsymbol{x}} \lambda(t, \boldsymbol{x})=\frac{r(t, \boldsymbol{x})}{a(t, \boldsymbol{x})},
$$

with

$$
\begin{aligned}
& a(t, \boldsymbol{x})=\int_{\mathbb{V}} \frac{M(\boldsymbol{v})}{G^{2}(t, \boldsymbol{x}, \boldsymbol{v})} d \boldsymbol{v}, \\
& \boldsymbol{B}(t, \boldsymbol{x})=\int_{\mathbb{V}} \frac{\boldsymbol{v} M(\boldsymbol{v})}{G^{2}(t, \boldsymbol{x}, \boldsymbol{v})} d \boldsymbol{v}, \\
& r(t, \boldsymbol{x})=\int_{\mathbb{V}} \frac{M(\boldsymbol{v})\left(\partial_{t} G(t, \boldsymbol{x}, \boldsymbol{v})+\boldsymbol{v} \cdot \nabla_{x} G(t, \boldsymbol{x}, \boldsymbol{v})\right)}{G^{3}(t, \boldsymbol{x}, \boldsymbol{v})} d \boldsymbol{v} .
\end{aligned}
$$

Proposition 2.2. Under the same assumptions in Theorem 2.1 and assume Sing $(M)=\emptyset, \psi^{2}(t, \boldsymbol{x}, \boldsymbol{v})$ is given by

$$
\begin{aligned}
\psi^{2}(t, \boldsymbol{x}, \boldsymbol{v}) & =\beta(t, \boldsymbol{x})-\frac{\partial_{t} \psi^{1}(t, \boldsymbol{x}, \boldsymbol{v})+\boldsymbol{v} \cdot \nabla_{x} \psi^{1}(t, \boldsymbol{x}, \boldsymbol{v})}{G(t, \boldsymbol{x}, \boldsymbol{v}))} \\
& =\beta(t, \boldsymbol{x})-\frac{\partial_{t} G(t, \boldsymbol{x}, \boldsymbol{v})+\boldsymbol{v} \cdot \nabla_{x} G(t, \boldsymbol{x}, \boldsymbol{v})}{G^{2}(t, \boldsymbol{x}, \boldsymbol{v})}+\frac{\partial_{t} \lambda(t, \boldsymbol{x})+\boldsymbol{v} \cdot \nabla_{x} \lambda(t, \boldsymbol{x})}{G(t, \boldsymbol{x}, \boldsymbol{v})},
\end{aligned}
$$

where $G$ is given by (14), and $\beta(t, \boldsymbol{x})$ satisfies the following transport equation,

$$
\partial_{t} \beta(t, \boldsymbol{x})+\frac{\boldsymbol{B}(t, \boldsymbol{x})}{a(t, \boldsymbol{x})} \cdot \nabla_{\boldsymbol{x}} \beta(t, \boldsymbol{x})=\frac{R(t, \boldsymbol{x})}{a(t, \boldsymbol{x})},
$$


with $\{a(t, \boldsymbol{x}), \boldsymbol{B}(t, \boldsymbol{x})\}$ given by 16 and

$$
\begin{aligned}
R(t, \boldsymbol{x})= & \int_{\mathbb{V}} \frac{M(\boldsymbol{v})\left[\partial_{t}^{2} G(t, \boldsymbol{x} \cdot \boldsymbol{v})+2 \boldsymbol{v} \cdot \nabla_{x} \partial_{t} G(t, \boldsymbol{x} \cdot \boldsymbol{v})+\boldsymbol{v} \cdot D_{x}^{2} G(t, \boldsymbol{x} \cdot \boldsymbol{v}) \cdot \boldsymbol{v}\right]}{G^{4}(t, \boldsymbol{x} \cdot \boldsymbol{v})} d \boldsymbol{v} \\
& -\int_{\mathbb{V}} \frac{M(\boldsymbol{v})\left[\partial_{t}^{2} \lambda(t, \boldsymbol{x})+2 \boldsymbol{v} \cdot \nabla_{x} \partial_{t} \lambda(t, \boldsymbol{x})+\boldsymbol{v} \cdot D_{x}^{2} \lambda(t, \boldsymbol{x}) \cdot \boldsymbol{v}\right]}{G^{3}(t, \boldsymbol{x} \boldsymbol{v})} d \boldsymbol{v} \\
& -3 \int_{\mathbb{V}} \frac{M(\boldsymbol{v})\left[\partial_{t} G(t, \boldsymbol{x} \boldsymbol{v})+\boldsymbol{v} \cdot \nabla_{x} G(t, \boldsymbol{x} \cdot \boldsymbol{v})\right]^{2}}{G^{5}(t, \boldsymbol{x} \cdot \boldsymbol{v})} d \boldsymbol{v}-\int_{\mathbb{V}} \frac{M(\boldsymbol{v})\left[\partial_{t} \lambda(t, \boldsymbol{x})+\boldsymbol{v} \cdot \nabla_{x} \lambda(t, \boldsymbol{x})\right]^{2}}{G^{3}(t, \boldsymbol{x} \boldsymbol{v})} d \boldsymbol{v} \\
& +3 \int_{\mathbb{V}} \frac{M(\boldsymbol{v})\left[\partial_{t} G(t, \boldsymbol{x} \boldsymbol{v})+\boldsymbol{v} \cdot \nabla_{x} G(t, \boldsymbol{x} \boldsymbol{v})\right]\left[\partial_{t} \lambda(t, \boldsymbol{x})+\boldsymbol{v} \cdot \nabla_{x} \lambda(t, \boldsymbol{x})\right]}{G^{4}(t, \boldsymbol{x}, \boldsymbol{v})} d \boldsymbol{v} .
\end{aligned}
$$

Proof of Proposition 2.1. Substituting the HC transformation (3) into equation (1) yields the following equation for $\psi^{\epsilon}$,

$$
1-\partial_{t} \psi^{\epsilon}(t, \mathbf{x}, \mathbf{v})-\mathbf{v} \cdot \nabla_{\mathbf{x}} \psi^{\epsilon}(t, \mathbf{x}, \mathbf{v})=\int_{\mathbb{V}} M\left(\mathbf{v}^{\prime}\right) \exp \left(\frac{\psi^{\epsilon}(t, \mathbf{x}, \mathbf{v})-\psi^{\epsilon}\left(t, \mathbf{x}, \mathbf{v}^{\prime}\right)}{\epsilon}\right) d \mathbf{v}^{\prime}
$$

From equation 20, we have

$$
\frac{M(\mathbf{v})}{1-\partial_{t} \psi^{\epsilon}(t, \mathbf{x}, \mathbf{v})-\mathbf{v} \cdot \nabla_{\mathbf{x}} \psi^{\epsilon}(t, \mathbf{x}, \mathbf{v})}=\frac{M(\mathbf{v}) \exp \left(-\frac{\psi^{\epsilon}(t, \mathbf{x}, \mathbf{v})}{\epsilon}\right)}{\int_{\mathbb{V}} M\left(\mathbf{v}^{\prime}\right) \exp \left(-\frac{\psi^{\epsilon}\left(t, \mathbf{x}, \mathbf{v}^{\prime}\right)}{\epsilon}\right) d \mathbf{v}^{\prime}}=\frac{f^{\epsilon}(t, \mathbf{x}, \mathbf{v})}{\int_{\mathbb{V}} f^{\epsilon}\left(t, \mathbf{x}, \mathbf{v}^{\prime}\right) d \mathbf{v}^{\prime}}
$$

Taking integration with respect to $\mathbf{v}$ on both sides of the above equation yields

$$
\int_{\mathbb{V}} \frac{M(\mathbf{v})}{1-\partial_{t} \psi^{\epsilon}(t, \mathbf{x}, \mathbf{v})-\mathbf{v} \cdot \nabla_{\mathbf{x}} \psi^{\epsilon}(t, \mathbf{x}, \mathbf{v})} d \mathbf{v}=1
$$

By subtracting equation 10 from equation 21, we have

$$
\int_{\mathbb{V}} \frac{M(\mathbf{v})\left(\partial_{t} \psi^{1, \epsilon}+\mathbf{v} \cdot \nabla_{\mathbf{x}} \psi^{1, \epsilon}\right)}{\left(1-\partial_{t} \psi^{\epsilon}-\mathbf{v} \cdot \nabla_{\mathbf{x}} \psi^{\epsilon}\right)\left(1-\partial_{t} \psi^{0}-\mathbf{v} \cdot \nabla_{\mathbf{x}} \psi^{0}\right)} d \mathbf{v}=0
$$

where $\psi^{1, \epsilon}=\psi^{1}+\epsilon \psi^{2}+\epsilon^{2} \psi^{3}+\cdots=\sum_{k=1}^{\infty} \epsilon^{k-1} \psi^{k}$. And from equation 20, we have

$$
\left[\left(1-\partial_{t} \psi^{0}-\mathbf{v} \cdot \nabla_{\mathbf{x}} \psi^{0}\right)+\epsilon\left(-\partial_{t} \psi^{1, \epsilon}-\mathbf{v} \cdot \nabla_{\mathbf{x}} \psi^{1, \epsilon}\right)\right] \exp \left(-\psi^{1, \epsilon}(t, \mathbf{x}, \mathbf{v})\right)=\int_{\mathbb{V}} M\left(\mathbf{v}^{\prime}\right) \exp \left(-\psi^{1, \epsilon}\left(t, \mathbf{x}, \mathbf{v}^{\prime}\right)\right) d \mathbf{v}^{\prime}
$$

Letting $\epsilon \rightarrow 0$ in equations 23 and 22 yields

$$
\left[1-\partial_{t} \psi^{0}(t, \mathbf{x})-\mathbf{v} \cdot \nabla_{\mathbf{x}} \psi^{0}(t, \mathbf{x})\right] \exp \left(-\psi^{1}(t, \mathbf{x}, \mathbf{v})\right)=\int_{\mathbb{V}} M\left(\mathbf{v}^{\prime}\right) \exp \left(-\psi^{1}\left(t, \mathbf{x}, \mathbf{v}^{\prime}\right)\right) d \mathbf{v}^{\prime}
$$


and

$$
\int_{\mathbb{V}} \frac{M(\mathbf{v})\left(\partial_{t} \psi^{1}(t, \mathbf{x}, \mathbf{v})+\mathbf{v} \cdot \nabla_{\mathbf{x}} \psi^{1}(t, \mathbf{x}, \mathbf{v})\right)}{\left(1-\partial_{t} \psi^{0}(t, \mathbf{x})-\mathbf{v} \cdot \nabla_{\mathbf{x}} \psi^{0}(t, \mathbf{x})\right)^{2}} d \mathbf{v}=0
$$

respectively.

By taking the logarithm on both sides of (24), and defining

$$
\lambda(t, \mathbf{x}) \equiv \log \left(\int_{\mathbb{V}} M\left(\mathbf{v}^{\prime}\right) \exp \left(-\psi^{1}\left(t, \mathbf{x}, \mathbf{v}^{\prime}\right)\right) d \mathbf{v}^{\prime}\right),
$$

we have the formula $(13)$. Then by substituting the formula $(13)$ into equation 25$)$, we have the transport equation (15) for $\lambda(t, \mathbf{x})$.

Proof of Proposition 2.2. From equation 22), we have

$$
\int_{\mathbb{V}} \frac{M(\mathbf{v})\left[\left(\partial_{t} \psi^{1}+\mathbf{v} \cdot \nabla_{\mathbf{x}} \psi^{1}\right)+\epsilon\left(\partial_{t} \psi^{2, \epsilon}+\mathbf{v} \cdot \nabla_{\mathbf{x}} \psi^{2, \epsilon}\right)\right]}{\left(1-\partial_{t} \psi^{\epsilon}-\mathbf{v} \cdot \nabla_{\mathbf{x}} \psi^{\epsilon}\right) G(t, \mathbf{x}, \mathbf{v})} d \mathbf{v}=0,
$$

with $\psi^{2, \epsilon}=\psi^{2}+\epsilon \psi^{3}+\epsilon^{2} \psi^{4}+\cdots=\sum_{k=2}^{\infty} \epsilon^{k-2} \psi^{k}$. From equation 26) and with the following Taylor expansion,

$$
\begin{aligned}
\frac{1}{1-\partial_{t} \psi^{\epsilon}-\mathbf{v} \cdot \nabla_{\mathbf{x}} \psi^{\epsilon}} & =\frac{1}{G(t, \mathbf{x}, \mathbf{v})-\epsilon\left(\partial_{t} \psi^{1}+\mathbf{v} \cdot \nabla_{\mathbf{x}} \psi^{1}\right)-\epsilon^{2}\left(\partial_{t} \psi^{2, \epsilon}+\mathbf{v} \cdot \nabla_{\mathbf{x}} \psi^{2, \epsilon}\right)} \\
& =\frac{1}{G(t, \mathbf{x}, \mathbf{v})}+\sum_{k=1}^{\infty} \frac{\left[\epsilon\left(\partial_{t} \psi^{1}+\mathbf{v} \cdot \nabla_{\mathbf{x}} \psi^{1}\right)+\epsilon^{2}\left(\partial_{t} \psi^{2, \epsilon}+\mathbf{v} \cdot \nabla_{\mathbf{x}} \psi^{2, \epsilon}\right)\right]^{k}}{G^{k+1}(t, \mathbf{x}, \mathbf{v})} \\
& =\frac{1}{G(t, \mathbf{x}, \mathbf{v})}+\frac{\epsilon\left(\partial_{t} \psi^{1}+\mathbf{v} \cdot \nabla_{\mathbf{x}} \psi^{1}\right)}{G^{2}(t, \mathbf{x}, \mathbf{v})}+O\left(\epsilon^{2}\right),
\end{aligned}
$$

we have

$$
\begin{aligned}
& \int_{\mathbb{V}} \frac{M(\mathbf{v})\left(\partial_{t} \psi^{1}+\mathbf{v} \cdot \nabla_{\mathbf{x}} \psi^{1}\right)}{G^{2}(t, \mathbf{x}, \mathbf{v})} d \mathbf{v}+\epsilon \int_{\mathbb{V}} \frac{M(\mathbf{v})\left(\partial_{t} \psi^{1}+\mathbf{v} \cdot \nabla_{\mathbf{x}} \psi^{1}\right)^{2}}{G^{3}(t, \mathbf{x}, \mathbf{v})} d \mathbf{v} \\
& +\epsilon \int_{\mathbb{V}} \frac{M(\mathbf{v})\left(\partial_{t} \psi^{2, \epsilon}+\mathbf{v} \cdot \nabla_{\mathbf{x}} \psi^{2, \epsilon}\right)}{\left(1-\partial_{t} \psi^{\epsilon}-\mathbf{v} \cdot \nabla_{\mathbf{x}} \psi^{\epsilon}\right) G(t, \mathbf{x}, \mathbf{v})} d \mathbf{v}=O\left(\epsilon^{2}\right) .
\end{aligned}
$$

After canceling out the first term in equation (27) due to equation (25), we have

$$
\int_{\mathbb{V}} \frac{M(\mathbf{v})\left(\partial_{t} \psi^{1}+\mathbf{v} \cdot \nabla_{\mathbf{x}} \psi^{1}\right)^{2}}{G^{3}(t, \mathbf{x}, \mathbf{v})} d \mathbf{v}+\int_{\mathbb{V}} \frac{M(\mathbf{v})\left(\partial_{t} \psi^{2, \epsilon}+\mathbf{v} \cdot \nabla_{\mathbf{x}} \psi^{2, \epsilon}\right)}{\left(1-\partial_{t} \psi^{\epsilon}-\mathbf{v} \cdot \nabla_{\mathbf{x}} \psi^{\epsilon}\right) G(t, \mathbf{x}, \mathbf{v})} d \mathbf{v}=O(\epsilon)
$$

which implies

$$
\int_{\mathbb{V}} \frac{M(\mathbf{v})\left(\partial_{t} \psi^{2}+\mathbf{v} \cdot \nabla_{\mathbf{x}} \psi^{2}\right)}{G^{2}(t, \mathbf{x}, \mathbf{v})}=-\int_{\mathbb{V}} \frac{M(\mathbf{v})\left(\partial_{t} \psi^{1}+\mathbf{v} \cdot \nabla_{\mathbf{x}} \psi^{1}\right)^{2}}{G^{3}(t, \mathbf{x}, \mathbf{v})} d \mathbf{v},
$$

50 as $\epsilon \rightarrow 0$. 
From equation 20, we have

$$
\begin{aligned}
& \left(1-\partial_{t} \psi^{0}-\mathbf{v} \cdot \nabla_{\mathbf{x}} \psi^{0}\right)-\epsilon\left(\partial_{t} \psi^{1}+\mathbf{v} \cdot \nabla_{\mathbf{x}} \psi^{1}\right)-\epsilon^{2}\left(\partial_{t} \psi^{2, \epsilon}+\mathbf{v} \cdot \nabla_{\mathbf{x}} \psi^{2, \epsilon}\right) \\
& =\int_{\mathbb{V}} M\left(\mathbf{v}^{\prime}\right) \exp \left(\psi^{1}(t, \mathbf{x}, \mathbf{v})-\psi^{1}\left(t, \mathbf{x}, \mathbf{v}^{\prime}\right)+\epsilon\left(\psi^{2, \epsilon}(t, \mathbf{x}, \mathbf{v})-\psi^{2, \epsilon}\left(t, \mathbf{x}, \mathbf{v}^{\prime}\right)\right)\right) d \mathbf{v}^{\prime}
\end{aligned}
$$

which, along with equation (24), implies

$$
\begin{aligned}
& -\left(\partial_{t} \psi^{1}+\mathbf{v} \cdot \nabla_{\mathbf{x}} \psi^{1}\right)-\epsilon\left(\partial_{t} \psi^{2, \epsilon}+\mathbf{v} \cdot \nabla_{\mathbf{x}} \psi^{2, \epsilon}\right) \\
& =\int_{\mathbb{V}} M\left(\mathbf{v}^{\prime}\right) \exp \left(\psi^{1}(t, \mathbf{x}, \mathbf{v})-\psi^{1}\left(t, \mathbf{x}, \mathbf{v}^{\prime}\right)\right)\left(\frac{\exp \left(\epsilon\left[\psi^{2, \epsilon}(t, \mathbf{x}, \mathbf{v})-\psi^{2, \epsilon}\left(t, \mathbf{x}, \mathbf{v}^{\prime}\right)\right]\right)-1}{\epsilon}\right) d \mathbf{v}^{\prime}
\end{aligned}
$$

Letting $\epsilon \rightarrow 0$ and using equation (24) again, we have

$$
\begin{aligned}
& -\left(\partial_{t} \psi^{1}(t, \mathbf{x}, \mathbf{v})+\mathbf{v} \cdot \nabla_{\mathbf{x}} \psi^{1}(t, \mathbf{x}, \mathbf{v})\right) \\
& =\int_{\mathbb{V}} M\left(\mathbf{v}^{\prime}\right) \exp \left(\psi^{1}(t, \mathbf{x}, \mathbf{v})-\psi^{1}\left(t, \mathbf{x}, \mathbf{v}^{\prime}\right)\right)\left(\psi^{2}(t, \mathbf{x}, \mathbf{v})-\psi^{2}\left(t, \mathbf{x}, \mathbf{v}^{\prime}\right)\right) d \mathbf{v}^{\prime} \\
& \left(\partial_{t} \psi^{1}(t, \mathbf{x}, \mathbf{v})+\mathbf{v} \cdot \nabla_{\mathbf{x}} \psi^{1}(t, \mathbf{x}, \mathbf{v})\right) e^{-\psi^{1}(t, \mathbf{x}, \mathbf{v})}+\int_{\mathbb{V}} M\left(\mathbf{v}^{\prime}\right) \exp \left(-\psi^{1}\left(t, \mathbf{x}, \mathbf{v}^{\prime}\right)\right) d \mathbf{v}^{\prime} \psi^{2}(t, \mathbf{x}, \mathbf{v}) \\
& =\int_{\mathbb{V}} M\left(\mathbf{v}^{\prime}\right) \exp \left(-\psi^{1}\left(t, \mathbf{x}, \mathbf{v}^{\prime}\right)\right) \psi^{2}\left(t, \mathbf{x}, \mathbf{v}^{\prime}\right) d \mathbf{v}^{\prime}, \\
& \left(\partial_{t} \psi^{1}(t, \mathbf{x}, \mathbf{v})+\mathbf{v} \cdot \nabla_{\mathbf{x}} \psi^{1}(t, \mathbf{x}, \mathbf{v})\right) e^{-\psi^{1}(t, \mathbf{x}, \mathbf{v})}+e^{\lambda(t, \mathbf{x})} \psi^{2}(t, \mathbf{x}, \mathbf{v}) \\
& \quad=\int_{\mathbb{V}} M\left(\mathbf{v}^{\prime}\right) \exp \left(-\psi^{1}\left(t, \mathbf{x}, \mathbf{v}^{\prime}\right)\right) \psi^{2}\left(t, \mathbf{x}, \mathbf{v}^{\prime}\right) d \mathbf{v}^{\prime} \\
& \frac{\partial_{t} \psi^{1}(t, \mathbf{x}, \mathbf{v})+\mathbf{v} \cdot \nabla_{\mathbf{x}} \psi^{1}(t, \mathbf{x}, \mathbf{v})}{G(t, \mathbf{x}, \mathbf{v})} e^{\lambda(t, \mathbf{x})}+e^{\lambda(t, \mathbf{x})} \psi^{2}(t, \mathbf{x}, \mathbf{v}) \\
& \quad=\int_{\mathbb{V}} M\left(\mathbf{v}^{\prime}\right) \exp \left(-\psi^{1}\left(t, \mathbf{x}, \mathbf{v}^{\prime}\right)\right) \psi^{2}\left(t, \mathbf{x}, \mathbf{v}^{\prime}\right) d \mathbf{v}^{\prime} \\
& \frac{\partial_{t} \psi^{1}(t, \mathbf{x}, \mathbf{v})+\mathbf{v} \cdot \nabla_{\mathbf{x}} \psi^{1}(t, \mathbf{x}, \mathbf{v})}{G(t, \mathbf{x}, \mathbf{v})}+\psi^{2}(t, \mathbf{x}, \mathbf{v}) \\
& =e^{-\lambda(t, \mathbf{x})} \int_{\mathbb{V}} M\left(\mathbf{v}^{\prime}\right) \exp \left(-\psi^{1}\left(t, \mathbf{x}, \mathbf{v}^{\prime}\right)\right) \psi^{2}\left(t, \mathbf{x}, \mathbf{v}^{\prime}\right) d \mathbf{v}^{\prime}
\end{aligned}
$$

By defining

$$
\beta(t, \mathbf{x}) \equiv e^{-\lambda(t, \mathbf{x})} \int_{\mathbb{V}} M\left(\mathbf{v}^{\prime}\right) \exp \left(-\psi^{1}\left(t, \mathbf{x}, \mathbf{v}^{\prime}\right)\right) \psi^{2}\left(t, \mathbf{x}, \mathbf{v}^{\prime}\right) d \mathbf{v}^{\prime},
$$

we have the formula (17). By substituting the formula (17) into equation (28) and after careful calculations, we have the transport equation 18 for $\beta(t, \mathbf{x})$.

Remark 1. With $\lambda(t, \boldsymbol{x})$ and $\beta(t, \boldsymbol{x})$ obtained from equations (15) and 18 respectively, we can compute $\psi^{1}(t, \boldsymbol{x}, \boldsymbol{v})$ and $\psi^{2}(t, \boldsymbol{x}, \boldsymbol{v})$ through formulae (13) and (17) respectively. The above derivations can be repeated 
to derive a sequence of equations such as equations (15) and (18) whose solutions can be used to compute the terms $\left\{\psi^{k}\right\}_{k=1}^{\infty}$ in the expansion (5). Higher order terms depend on lower order terms. In this work, we focus on $\psi^{0}, \psi^{1}$ and $\psi^{2}$.

\subsection{Formulations for Numerical Implementations}

We further simplify the transport equations 15$)$ and $(18)$ for numerical implementations by removing the time derivatives in the definitions of $r(t, \mathbf{x})$ and $R(t, \mathbf{x})$. That is, we can evaluate $G, \partial_{t} G, \nabla_{\mathbf{x}} G, \partial_{t}^{2} G, \nabla_{\mathbf{x}} \partial_{t} G$, $D_{\mathbf{x}}^{2} G, \partial_{t} \lambda, \partial_{t}^{2} \lambda$, and $\nabla_{\mathbf{x}} \partial_{t} \lambda$ in formulae 16 and 190 with only function values and spatial derivatives of $\left\{\psi^{0}, \lambda, \beta\right\}, H\left(\nabla_{\mathbf{x}} \psi^{0}\right), \nabla_{\mathbf{p}} H\left(\nabla_{\mathbf{x}} \psi^{0}\right)$, and $D_{\mathbf{p}}^{2} H\left(\nabla_{\mathbf{x}} \psi^{0}\right)$.

From the effective HJ equation (8), we have

$$
\begin{aligned}
\partial_{t} \psi^{0}(t, \mathbf{x}) & =-H\left(\nabla_{\mathbf{x}} \psi^{0}(t, \mathbf{x})\right) \\
\nabla_{\mathbf{x}} \partial_{t} \psi^{0}(t, \mathbf{x}) & =-\nabla_{\mathbf{p}} H\left(\nabla_{\mathbf{x}} \psi^{0}\right) \cdot D_{\mathbf{x}}^{2} \psi^{0}(t, \mathbf{x}) .
\end{aligned}
$$

By the definition of $G(t, \mathbf{x}, \mathbf{v})$ in (14), we have

$$
\begin{aligned}
G(t, \mathbf{x}, \mathbf{v})= & 1+H\left(\nabla_{\mathbf{x}} \psi^{0}(t, \mathbf{x})\right)-\mathbf{v} \cdot \nabla_{\mathbf{x}} \psi^{0}(t, \mathbf{x}) \\
\nabla_{\mathbf{x}} G(t, \mathbf{x}, \mathbf{v})= & \left(\nabla_{\mathbf{p}} H\left(\nabla_{\mathbf{x}} \psi^{0}\right)-\mathbf{v}\right) \cdot D_{\mathbf{x}}^{2} \psi^{0}, \\
\partial_{t} G(t, \mathbf{x}, \mathbf{v})= & \nabla_{\mathbf{p}} H\left(\nabla_{\mathbf{x}} \psi^{0}\right) \cdot \nabla_{\mathbf{x}} \partial_{t} \psi^{0}(t, \mathbf{x})-\mathbf{v} \cdot \nabla_{\mathbf{x}} \partial_{t} \psi^{0}(t, \mathbf{x}) \\
= & -\left(\nabla_{\mathbf{p}} H\left(\nabla_{\mathbf{x}} \psi^{0}\right)-\mathbf{v}\right) \cdot D_{\mathbf{x}}^{2} \psi^{0} \cdot \nabla_{\mathbf{p}} H\left(\nabla_{\mathbf{x}} \psi^{0}\right), \\
\partial_{t}^{2} G(t, \mathbf{x}, \mathbf{v})= & -\left(2 \nabla_{\mathbf{p}} H\left(\nabla_{\mathbf{x}} \psi^{0}\right)-\mathbf{v}\right) \cdot D_{\mathbf{x}}^{2} \psi^{0} \cdot\left[D_{\mathbf{p}}^{2} H\left(\nabla_{\mathbf{x}} \psi^{0}\right) \cdot \nabla_{\mathbf{x}} \partial_{t} \psi^{0}\right] \\
= & -\left(2 \nabla_{\mathbf{p}} H\left(\nabla_{\mathbf{x}} \psi^{0}\right)-\mathbf{v}\right) \cdot D_{\mathbf{x}}^{2} \psi^{0} \cdot\left[D_{\mathbf{p}}^{2} H\left(\nabla_{\mathbf{x}} \psi^{0}\right) \cdot\left(\nabla_{\mathbf{p}} H\left(\nabla_{\mathbf{x}} \psi^{0}\right) \cdot D_{\mathbf{x}}^{2} \psi^{0}\right)\right], \\
\nabla_{\mathbf{x}} \partial_{t} G(t, \mathbf{x}, \mathbf{v})= & -\left(D_{\mathbf{p}}^{2} H\left(\nabla_{\mathbf{x}} \psi^{0}\right) \cdot\left(\nabla_{\mathbf{p}} H\left(\nabla_{\mathbf{x}} \psi^{0}\right) \cdot D_{\mathbf{x}}^{2} \psi^{0}\right)\right) \cdot D_{\mathbf{x}}^{2} \psi^{0} \\
& -\left(\nabla_{\mathbf{p}} H\left(\nabla_{\mathbf{x}} \psi^{0}\right)-\mathbf{v}\right) \cdot\left[D_{\mathbf{x}}^{2} \psi^{0} \cdot D_{\mathbf{p}}^{2} H\left(\nabla_{\mathbf{x}} \psi^{0}\right) \cdot D_{\mathbf{x}}^{2} \psi^{0}+\nabla_{\mathbf{p}} H\left(\nabla_{\mathbf{x}} \psi^{0}\right) \cdot \nabla_{\mathbf{x}} D_{\mathbf{x}}^{2} \psi^{0}\right], \\
D_{\mathbf{x}}^{2} G(t, \mathbf{x}, \mathbf{v})= & D_{\mathbf{x}}^{2} \psi^{0} \cdot D_{\mathbf{p}}^{2} H\left(\nabla_{\mathbf{x}} \psi^{0}\right) \cdot D_{\mathbf{x}}^{2} \psi^{0}-\mathbf{v} \cdot \nabla_{\mathbf{x}} D_{\mathbf{x}}^{2} \psi^{0} .
\end{aligned}
$$

From the transport equation 15 , we have

$$
\begin{aligned}
& \partial_{t} \lambda(t, \mathbf{x})=\frac{r(t, \mathbf{x})}{a(t, \mathbf{x})}-\frac{\mathbf{B}(t, \mathbf{x})}{a(t, \mathbf{x})} \cdot \nabla_{\mathbf{x}} \lambda(t, \mathbf{x}), \\
& \nabla_{\mathbf{x}} \partial_{t} \lambda(t, \mathbf{x})=\frac{a \nabla_{\mathbf{x}} r-r \nabla_{\mathbf{x}} a}{a^{2}}-\frac{a \nabla_{\mathbf{x}} \mathbf{B}-\mathbf{B} \otimes \nabla_{\mathbf{x}} a}{a^{2}} \cdot \nabla_{\mathbf{x}} \lambda-\frac{\mathbf{B}(t, \mathbf{x})}{a} \cdot D_{\mathbf{x}}^{2} \lambda,
\end{aligned}
$$


and

$$
\begin{aligned}
\partial_{t}^{2} \lambda(t, \mathbf{x})= & \frac{a \partial_{t} r-r \partial_{t} a}{a^{2}}-\frac{a \partial_{t} \mathbf{B}-\mathbf{B} \partial_{t} a}{a^{2}} \cdot \nabla_{\mathbf{x}} \lambda-\frac{\mathbf{B}}{a} \cdot \nabla_{\mathbf{x}} \partial_{t} \lambda \\
= & \frac{a \partial_{t} r-r \partial_{t} a}{a^{2}}-\frac{a \partial_{t} \mathbf{B}-\mathbf{B} \partial_{t} a}{a^{2}} \cdot \nabla_{\mathbf{x}} \lambda \\
& -\frac{\mathbf{B}}{a} \cdot\left[\frac{a \nabla_{\mathbf{x}} r-r \nabla_{\mathbf{x}} a}{a^{2}}-\frac{a \nabla_{\mathbf{x}} \mathbf{B}-\mathbf{B} \otimes \nabla_{\mathbf{x}} a}{a^{2}} \cdot \nabla_{\mathbf{x}} \lambda-\frac{\mathbf{B}}{a} \cdot D_{\mathbf{x}}^{2} \lambda\right] .
\end{aligned}
$$

In order to evaluate $\partial_{t} a, \partial_{t} \mathbf{B}, \partial_{t} r, \nabla_{\mathbf{x}} a, \nabla_{\mathbf{x}} \mathbf{B}$ and $\nabla_{\mathbf{x}} r$ in the above formulae, we have

$$
\begin{aligned}
\partial_{t} a(t, \mathbf{x}) & =-2 \int_{\mathbb{V}} \frac{M(\mathbf{v}) \partial_{t} G(t, \mathbf{x}, \mathbf{v})}{G^{3}(t, \mathbf{x}, \mathbf{v})} d \mathbf{v} \\
\nabla_{\mathbf{x}} a(t, \mathbf{x}) & =-2 \int_{\mathbb{V}} \frac{M(\mathbf{v}) \nabla_{\mathbf{x}} G(t, \mathbf{x}, \mathbf{v})}{G^{3}(t, \mathbf{x}, \mathbf{v})} d \mathbf{v} \\
\partial_{t} \mathbf{B}(t, \mathbf{x}) & =-2 \int_{\mathbb{V}} \frac{\mathbf{v} M(\mathbf{v}) \partial_{t} G(t, \mathbf{x}, \mathbf{v})}{G^{3}(t, \mathbf{x}, \mathbf{v})} d \mathbf{v} \\
\nabla_{\mathbf{x}} \mathbf{B}(t, \mathbf{x}) & =-2 \int_{\mathbb{V}} \frac{\mathbf{v} M(\mathbf{v}) \otimes \nabla_{\mathbf{x}} G(t, \mathbf{x}, \mathbf{v})}{G^{3}(t, \mathbf{x}, \mathbf{v})} d \mathbf{v} \\
\partial_{t} r(t, \mathbf{x}) & =-2 \int_{\mathbb{V}} \frac{M(\mathbf{v})\left[\left(\partial_{t}^{2} G+\mathbf{v} \cdot \nabla_{\mathbf{x}} \partial_{t} G\right) G^{3}-3\left(\partial_{t} G+\mathbf{v} \cdot \nabla_{\mathbf{x}} G\right) G^{2} \partial_{t} G\right]}{G^{6}(t, \mathbf{x}, \mathbf{v})} d \mathbf{v}, \\
\nabla_{\mathbf{x}} r(t, \mathbf{x}) & =-2 \int_{\mathbb{V}} \frac{M(\mathbf{v})\left[\left(\nabla_{\mathbf{x}} \partial_{t} G+\mathbf{v} \cdot D_{\mathbf{x}}^{2} G\right) G^{3}-3\left(\partial_{t} G+\mathbf{v} \cdot \nabla_{\mathbf{x}} G\right) G^{2} \nabla_{\mathbf{x}} G\right]}{G^{6}(t, \mathbf{x}, \mathbf{v})} d \mathbf{v},
\end{aligned}
$$

where the evaluations of $G, \partial_{t} G, \nabla_{\mathbf{x}} G, \partial_{t}^{2} G, \nabla_{\mathbf{x}} \partial_{t} G$, and $D_{\mathbf{x}}^{2} G$ can be done by previous formulae.

Furthermore, from equations (11) and [12), we will evaluate $\nabla_{\mathbf{p}} H(\mathbf{p})$ and $D_{\mathbf{p}}^{2} H(\mathbf{p})$ as

$$
\begin{aligned}
\nabla_{\mathbf{p}} H(\mathbf{p}) & =\frac{\mathbf{B}}{a} \\
D_{\mathbf{p}}^{2} H(\mathbf{p}) & =\frac{2}{a} \int_{\mathbb{V}} \frac{M(\mathbf{v})\left[\left(\nabla_{\mathbf{p}} H(\mathbf{p})-\mathbf{v}\right) \otimes\left(\nabla_{\mathbf{p}} H(\mathbf{p})-\mathbf{v}\right)\right]}{(1+H(\mathbf{p})-\mathbf{v} \cdot \mathbf{p})^{3}} d \mathbf{v} \\
& =\frac{2}{a} \int_{\mathbb{V}} \frac{M(\mathbf{v})[(\mathbf{B} / a-\mathbf{v}) \otimes(\mathbf{B} / a-\mathbf{v})]}{(1+H(\mathbf{p})-\mathbf{v} \cdot \mathbf{p})^{3}} d \mathbf{v}
\end{aligned}
$$

\section{Numerical Schemes}

With the formulations derived in Section $2, \psi^{0}$ is determined by equations (8) and (9), $\psi^{1}$ is obtained through the solution $\lambda$ of equation (15) and $\psi^{0}$ with formula 137 , and $\psi^{2}$ is obtained through the solution $\beta$ of equation (18), $\lambda$ and $\psi^{0}$ with formula (17). Once they are computed, $f^{\epsilon}$ is approximated by formula (6).

From the above derivations, we note that for solving the transport equation (15) for $\lambda(t, \mathbf{x})$, the first and second spatial derivatives of $\psi^{0}$ and $\nabla_{\mathbf{p}} H\left(\nabla_{\mathbf{x}} \psi^{0}\right)$ are involved; and for solving the transport equation (18) for $\beta(t, \mathbf{x})$, the first, second and third derivatives of $\psi^{0}$, the first and second derivatives of $\lambda$, and 
$\left\{\nabla_{\mathbf{p}} H\left(\nabla_{\mathbf{x}} \psi^{0}\right), D_{\mathbf{p}}^{2} H\left(\nabla_{\mathbf{x}} \psi^{0}\right)\right\}$ are involved. Hence, higher order schemes are required. For instance, in order to get first order accurate $\beta$, third order accurate $\lambda$ and fifth order accurate $\psi^{0}$ are needed. This is similar to the situation in computational geometrical optics with WKB approximations as explained in [22.

In this section, we present high order numerical schemes for solving equations (8), 15) and (18).

\subsection{High Order Schemes}

For notational simplicity, we present the schemes in 2-D for a generic equation with the form,

$$
\partial_{t} \phi(t, \mathbf{x})+H\left(t, \mathbf{x}, \nabla_{\mathbf{x}} \phi(t, \mathbf{x})\right)=s(t, \mathbf{x})
$$

Assume the computational domain is $\Omega \equiv\left\{\mathbf{x} \equiv(x, y): x_{\min } \leq x \leq x_{\max }, y_{\min } \leq y \leq y_{\max }\right\}$, where periodic boundary conditions in $(x, y)$ are imposed, and the velocity domain is $\mathbb{V} \equiv\left\{\mathbf{v} \equiv(u, v): u_{\min } \leq\right.$ $\left.u \leq u_{\max }, v_{\min } \leq v \leq v_{\max }\right\}$ with $u_{\min }=-u_{\max }$ and $v_{\min }=-v_{\max }$. The domain $\Omega$ is discretized and covered by a uniform mesh $\Omega^{h} \equiv\left\{\left(x_{i}, y_{j}\right): x_{i}=x_{\min }+i \Delta x, y_{j}=y_{\min }+j \Delta y ; 0 \leq i \leq N_{x}, 0 \leq j \leq N_{y}\right\}$ with mesh sizes $\Delta x=\left(x_{\max }-x_{\min }\right) / N_{x}$, and $\Delta y=\left(y_{\max }-y_{\min }\right) / N_{y}$. And the time step size is denoted as $\Delta t$. For mesh sizes $\Delta x, \Delta y, \Delta t, \phi_{i, j}^{k}$ will denote a numerical approximation to the viscosity solution $\phi\left(t^{k}, x_{i}, y_{j}\right)=\phi\left(k \Delta t, x_{\min }+i \Delta x, y_{\min }+j \Delta y\right)$. Some standard notations will be used such as

$$
\Delta_{ \pm}^{x} \phi_{i, j}= \pm\left(\phi_{i \pm 1, j}-\phi_{i, j}\right), \text { and } \Delta_{ \pm}^{y} \phi_{i, j}= \pm\left(\phi_{i, j \pm 1}-\phi_{i, j}\right)
$$

The building block is the first order monotone scheme [7,

$$
\phi_{i, j}^{k+1}=\phi_{i, j}^{k}-\Delta t \hat{H}\left(\frac{\Delta_{+}^{x} \phi_{i, j}^{k}}{\Delta x}, \frac{\Delta_{-}^{x} \phi_{i, j}^{k}}{\Delta x}, \frac{\Delta_{+}^{y} \phi_{i, j}^{k}}{\Delta y}, \frac{\Delta_{-}^{y} \phi_{i, j}^{k}}{\Delta y}\right)+\Delta t s_{i, j}^{k},
$$

where $\hat{H}$ is a monotone numerical Hamiltonian consistent with $H$, i.e.,

$$
\hat{H}(p, p, q, q)=H(p, q),
$$

$\hat{H}$ is non-increasing in its first and third arguments and nondecreasing in the other two, and the time step size $\Delta t$ is chosen such that the Courant-Friedrichs-Lewy (CFL) condition is satisfied, i.e.,

$$
V_{\max }\left(\frac{\Delta t}{\Delta x}+\frac{\Delta t}{\Delta y}\right) \leq 1
$$


We consider the following monotone Godunov Hamiltonian $\hat{H}$ [1, 20],

$$
\hat{H}^{G}\left(p^{+}, p^{-}, q^{+}, q^{-}\right)=\operatorname{ext}_{p \in I\left(p^{-}, p^{+}\right)} \operatorname{ext}_{q \in I\left(q^{-}, q^{+}\right)} H(p, q),
$$

where

$$
\operatorname{ext}_{p \in I(a, b)}=\left\{\begin{array}{l}
\min _{a \leq p \leq b} \text { if } a \leq b \\
\max _{b \leq p \leq a} \text { if } a>b .
\end{array}\right.
$$

The Godunov Hamiltonian $\hat{H}^{G}$ is monotone for $A \leq p^{ \pm} \leq B, C \leq q^{ \pm} \leq D$, with $A, B, C$ and $D$ appropriate constants.

For high order schemes, we use the weighted essentially non-oscillatory (WENO) finite difference approximations to approximate $p^{ \pm}=\phi_{x}^{ \pm}, q^{ \pm}=\phi_{y}^{ \pm}$, and the Runge-Kutta (RK) procedures for time discretization.

For approximating $\phi_{x}^{ \pm}$and $\phi_{y}^{ \pm}$, we use fifth order WENO construction (WENO5) [16, 12, 23, i.e.,

$$
\begin{aligned}
\left(\phi_{x}^{-}\right)_{i, j} & =\frac{1}{12 \Delta x}\left(-\Delta_{+}^{x} \phi_{i-2, j}+7 \Delta_{+}^{x} \phi_{i-1, j}+7 \Delta_{+}^{x} \phi_{i, j}-\Delta_{+}^{x} \phi_{i+1, j}\right) \\
& -\Phi^{W E N O}\left(\frac{\Delta_{-}^{x} \Delta_{+}^{x} \phi_{i-2, j}}{\Delta x}, \frac{\Delta_{-}^{x} \Delta_{+}^{x} \phi_{i-1, j}}{\Delta x}, \frac{\Delta_{-}^{x} \Delta_{+}^{x} \phi_{i, j}}{\Delta x}, \frac{\Delta_{-}^{x} \Delta_{+}^{x} \phi_{i+1, j}}{\Delta x}\right),
\end{aligned}
$$

where

$$
\begin{aligned}
& \Phi^{W E N O}(a, b, c, d)=\frac{1}{3} \omega_{0}(a-2 b+c)+\frac{1}{6}\left(\omega_{2}-\frac{1}{2}\right)(b-2 c+d), \\
& \omega_{0}=\frac{\alpha_{0}}{\alpha_{0}+\alpha_{1}+\alpha_{2}}, \omega_{2}=\frac{\alpha_{2}}{\alpha_{0}+\alpha_{1}+\alpha_{2}}, \\
& \alpha_{0}=\frac{1}{\left(\epsilon_{0}+I S_{0}\right)^{2}}, \alpha_{1}=\frac{6}{\left(\epsilon_{0}+I S_{1}\right)^{2}}, \alpha_{2}=\frac{3}{\left(\epsilon_{0}+I S_{2}\right)^{2}}, \\
& I S_{0}=13(a-b)^{2}+3(a-3 b)^{2} \\
& I S_{1}=13(b-c)^{2}+3(b+c)^{2} \\
& I S_{2}=13(c-d)^{2}+3(3 c-d)^{2}
\end{aligned}
$$

and

$$
\begin{aligned}
\left(\phi_{x}^{+}\right)_{i, j} & =\frac{1}{12 \Delta x}\left(-\Delta_{+}^{x} \phi_{i-2, j}+7 \Delta_{+}^{x} \phi_{i-1, j}+7 \Delta_{+}^{x} \phi_{i, j}-\Delta_{+}^{x} \phi_{i+1, j}\right) \\
& -\Phi^{W E N O}\left(\frac{\Delta_{-}^{x} \Delta_{+}^{x} \phi_{i+2, j}}{\Delta x}, \frac{\Delta_{-}^{x} \Delta_{+}^{x} \phi_{i+1, j}}{\Delta x}, \frac{\Delta_{-}^{x} \Delta_{+}^{x} \phi_{i, j}}{\Delta x}, \frac{\Delta_{-}^{x} \Delta_{+}^{x} \phi_{i-1, j}}{\Delta x}\right) .
\end{aligned}
$$

WENO5 approximations for $\phi_{y}^{ \pm}$can be constructed similarly. $\epsilon_{0}$ is chosen as $10^{-13}$ to avoid division by zero. 
For the time discretization, we use the fifth order RK method by Lawson [15], i.e.,

$$
\begin{aligned}
K_{1} & =L\left(\phi^{k}\right) \\
K_{2} & =L\left(\phi^{k}+\Delta t a_{21} K_{1}\right), \\
K_{3} & =L\left(\phi^{k}+\Delta t\left(a_{31} K_{1}+a_{32} K_{2}\right)\right), \\
K_{4} & =L\left(\phi^{k}+\Delta t\left(a_{41} K_{1}+a_{42} K_{2}+a_{43} K_{3}\right)\right), \\
K_{5} & =L\left(\phi^{k}+\Delta t\left(a_{51} K_{1}+a_{52} K_{2}+a_{53} K_{3}+a_{54} K_{4}\right)\right), \\
K_{6} & =L\left(\phi^{k}+\Delta t\left(a_{61} K_{1}+a_{62} K_{2}+a_{63} K_{3}+a_{64} K_{4}+a_{65} K_{5}\right)\right), \\
\phi^{k+1} & =\phi^{k}+\Delta t\left(b_{1} K_{1}+b_{2} K_{2}+b_{3} K_{3}+b_{4} K_{4}+b_{5} K_{5}+b_{6} K_{6}\right),
\end{aligned}
$$

where

$$
\begin{gathered}
\left(\begin{array}{lllll}
a_{21} & & & \\
a_{31} & a_{32} & & & \\
a_{41} & a_{42} & a_{43} & & \\
a_{51} & a_{52} & a_{53} & a_{54} & \\
a_{61} & a_{62} & a_{63} & a_{64} & a_{65}
\end{array}\right)\left(\begin{array}{ccccc}
1 / 12 & & & \\
-1 / 8 & 3 / 8 & & & \\
3 / 5 & -9 / 10 & 4 / 5 & & \\
39 / 80 & -9 / 20 & 3 / 20 & 9 / 16 & \\
-59 / 35 & 66 / 35 & 48 / 35 & -12 / 7 & 8 / 7
\end{array}\right), \\
\left(\begin{array}{c}
b_{1} \\
b_{2} \\
b_{3} \\
b_{4} \\
b_{5} \\
b_{6}
\end{array}\right)=\left(\begin{array}{c}
7 / 90 \\
0 \\
32 / 90 \\
12 / 90 \\
32 / 90 \\
7 / 90
\end{array}\right),
\end{gathered}
$$

and $L(\phi)=-\hat{H}\left(\phi_{x}^{ \pm}, \phi_{y}^{ \pm}\right)+s(x, y)$.

With the numerical techniques presented above, the algorithm based on RK and WENO is summarized as:

\section{Algorithm 1. WENO-RK method}

- For $k=0,1,2, \ldots$

- at any node $\left(x_{i}, y_{j}\right)$,

* use the WENO constructions to approximate $\left(\phi_{x}^{ \pm}\right)_{i, j}$ and $\left(\phi_{y}^{ \pm}\right)_{i, j}$,

* use the RK procedures to obtain $\phi_{i, j}^{k+1}$.

$90 \quad$ We use Algorithm 1 to solve equations (8), 15 and (18) numerically. 


\subsection{Computation of $\psi^{0}$}

When applying Algorithm 1 to numerically solve the effective HJ equation (8), we need to evaluate the numerical Hamiltonian $\hat{H}$ and maintain the positivity of $\psi^{0}[17$.

Given $p, q$, the effective Hamiltonian $H(p, q)$, as well as the numerical Hamiltonian $\hat{H}^{G}\left(p^{+}, p^{-}, q^{+}, q^{-}\right)$, are determined implicitly by equation (9) which is a nonlinear function in $H$, i.e.,

$$
F(H(p, q)) \equiv \int_{v_{\min }}^{v_{\max }} \int_{u_{\min }}^{u_{\max }} \frac{M(u, v)}{1+H(p, q)-u p-v q} d u d v-1=0 .
$$

From Theorem 2.1. we know $H(p, q) \geq \max _{(u, v) \in \mathbb{V}}\{u p+v p-1\}, H(0,0)=0$, and $\nabla_{\mathbf{p}} H(0,0)=0$. From the convexity of $H$, we know $H(p, q) \geq 0$. Hence, we must find the solution of the nonlinear equation for $H(p, q) \in I_{H(p, q)} \equiv\left[\max \left\{0, \max _{(u, v) \in \mathbb{V}}\{u p+v p-1\}\right\}, \infty\right)$. Since

$$
F^{\prime}(H)=-\int_{v_{\min }}^{v_{\max }} \int_{u_{\min }}^{u_{\max }} \frac{M(u, v)}{(1+H-u p-v q)^{2}} d u d v<0,
$$

$F(H)$ is decreasing in $I_{H(p, q)}$, which implies the solution is unique in $I_{H(p, q)}$ [17. We use Newton's method to solve the nonlinear equation (9). Starting with an initial guess $H_{0}$, the method generates a sequence $\left\{H_{m}\right\}$ that converges to $H$ by

$$
H_{m}=H_{m-1}-\frac{F\left(H_{m-1}\right)}{F^{\prime}\left(H_{m-1}\right)}, m \geq 1
$$

Both $F(H)$ and $F^{\prime}(H)$ must be evaluated as integrals in the velocity variable $(u, v)$, for which we use Gauss quadrature rules, i.e.,

$$
\begin{aligned}
& F(H) \approx \sum_{s} \sum_{t} \frac{w_{s}^{u} w_{t}^{v}}{1+H-u_{s} p-v_{t} q}-1, \\
& F^{\prime}(H) \approx-\sum_{s} \sum_{t} \frac{w_{s}^{u} w_{t}^{v}}{\left(1+H-u_{s} p-v_{t} q\right)^{2}},
\end{aligned}
$$

where $\left\{w_{s}^{u}, u_{s}\right\}$ (respectively, $\left.\left\{w_{t}^{v}, v_{t}\right\}\right)$ are weights and abscissas of an appropriate Gauss quadrature rule on $\left[u_{\min }, u_{\max }\right]$ (respectively, $\left.\left[v_{\min }, v_{\max }\right]\right)$.

We summarize Newton's method for solving the nonlinear equation (9) [17].

Algorithm 2. Newton's method for equation (9)

1. Initial guess: set $H_{0} \geq \max \left\{0,|p| u_{\max }+|q| v_{\max }-1\right\}$.

2. Newton's iterations: for $m \geq 1$,

$$
H_{m}=\max \left\{H_{m-1}-\frac{F\left(H_{m-1}\right)}{F^{\prime}\left(H_{m-1}\right)}, 0,|p| u_{\max }+|q| v_{\max }-1\right\}
$$

where $F\left(H_{m-1}\right)$ and $F^{\prime}\left(H_{m-1}\right)$ are approximated by Gauss quadrature rules 32 . 
3. Termination: if $\left|F\left(H_{m}\right)\right|<\delta$ for some termination criterion $\delta>0$, or $m \geq M_{i t e r}$ for some maximum number of iterations $M_{i t e r}$, stop the iterations.

With Algorithm 2, the positivity of the effective Hamiltonian is maintained [17.

Therefore, when applying Algorithm 1 to solve the effective HJ equation (8), we need to use Algorithm 2 to compute the numerical Hamiltonian $\hat{H}$. Furthermore, in order to maintain the positivity of $\psi^{0}$, we set

$$
\psi_{i, j}^{0, k+1}=\max \left\{\psi_{i, j}^{0, k+1}, 0\right\}
$$

at the final stage of the RK procedure. This simple enforcement does not affect the order of accuracy of the WENO-RK method as proved in [17.

\subsection{Computation of $\psi^{1}$ and $\psi^{2}$}

We can compute $\psi^{1}(t, \mathbf{x}, \mathbf{v})$ with $\lambda(t, \mathbf{x})$ and $\psi^{0}(t, \mathbf{x})$ as in formula 13 , where $\lambda(t, \mathbf{x})$ is obtained by solving the transport equation (15) with Algorithm 1. When applying Algorithm 1 to solve equation (15), we compute the coefficients $a(t, \mathbf{x}), \mathbf{B}(t, \mathbf{x})$ and $r(t, \mathbf{x})$ with Gauss quadrature rules, i.e.,

$$
\begin{aligned}
& a(t, \mathbf{x}) \approx \sum_{s} \sum_{t} \frac{w_{s}^{u} w_{t}^{v}}{\left(1+H-u_{s} \psi_{x}^{0}-v_{t} \psi_{y}^{0}\right)^{2}}, \\
& \mathbf{B}(t, \mathbf{x}) \approx \sum_{s} \sum_{t} \frac{w_{s}^{u} w_{t}^{v}\left(u_{s}, v_{t}\right)}{\left(1+H-u_{s} \psi_{x}^{0}-v_{t} \psi_{y}^{0}\right)^{2}}, \\
& r(t, \mathbf{x}) \approx \sum_{s} \sum_{t} \frac{w_{s}^{u} w_{t}^{v}\left(-\left(\frac{\mathbf{B}(t, \mathbf{x})}{a(t, \mathbf{x})}-\left(u_{s}, v_{t}\right)\right) \cdot D_{\mathbf{x}}^{2} \psi^{0}(t, \mathbf{x}) \cdot\left(\frac{\mathbf{B}(t, \mathbf{x})}{a(t, \mathbf{x})}-\left(u_{s}, v_{t}\right)\right)\right)}{\left(1+H-u_{s} \psi_{x}^{0}-v_{t} \psi_{y}^{0}\right)^{3}} .
\end{aligned}
$$

We can compute $\psi^{2}(t, \mathbf{x}, \mathbf{v})$ with $\beta(t, \mathbf{x}), \lambda(t, \mathbf{x})$ and $\psi^{0}(t, \mathbf{x})$ as in formula (17), where $\beta(t, \mathbf{x})$ is obtained by solving the transport equation (18) with Algorithm 1 . When applying Algorithm 1 to solve equation (18), we compute the coefficients $a(t, \mathbf{x}), \mathbf{B}(t, \mathbf{x})$ and $R(t, \mathbf{x})$ with same Gauss quadrature rules as above.

\subsection{Approximation of $f^{\epsilon}$}

At a given time $t$, once $\psi^{0}(t, \mathbf{x}), \lambda(t, \mathbf{x})$ and $\beta(t, \mathbf{x})$ are computed, we can first compute $G(t, \mathbf{x}, \mathbf{v})=$ $1-\partial_{t} \psi^{0}(t, \mathbf{x})-\mathbf{v} \cdot \nabla_{\mathbf{x}} \psi^{0}(t, \mathbf{x})=1+H\left(\nabla_{\mathbf{x}} \psi^{0}(t, \mathbf{x})\right)-\mathbf{v} \cdot \nabla_{\mathbf{x}} \psi^{0}(t, \mathbf{x})$ with $H\left(\nabla_{\mathbf{x}} \psi^{0}(t, \mathbf{x})\right)$ computed by Algorithm 2, then obtain $\psi^{1}(t, \mathbf{x}, \mathbf{v})$ and $\psi^{2}(t, \mathbf{x}, \mathbf{v})$ with formulae 13) and (17) respectively, and finally approximate $f^{\epsilon}(t, \mathbf{x}, \mathbf{v})$ with formula (6). 
We know

$$
\begin{aligned}
f^{\epsilon}(t, \mathbf{x}, \mathbf{v}) & =M(\mathbf{v}) \exp \left(-\frac{\psi^{\epsilon}(t, \mathbf{x}, \mathbf{v})}{\epsilon}\right) \\
& =M(\mathbf{v}) \exp \left(-\frac{\psi^{0}(t, \mathbf{x})}{\epsilon}-\psi^{1}(t, \mathbf{x}, \mathbf{v})-\epsilon \psi^{2}(t, \mathbf{x}, \mathbf{v})+O\left(\epsilon^{2}\right)\right) \\
& =M(\mathbf{v}) \exp \left(-\frac{\psi^{0}(t, \mathbf{x})}{\epsilon}-\psi^{1}(t, \mathbf{x}, \mathbf{v})-\epsilon \psi^{2}(t, \mathbf{x}, \mathbf{v})\right)\left(1+O\left(\epsilon^{2}\right)\right)
\end{aligned}
$$

which implies

$$
\left|\frac{f^{\epsilon}(t, \mathbf{x}, \mathbf{v})-M(\mathbf{v}) \exp \left(-\frac{\psi^{0}(t, \mathbf{x})}{\epsilon}-\psi^{1}(t, \mathbf{x}, \mathbf{v})-\epsilon \psi^{2}(t, \mathbf{x}, \mathbf{v})\right)}{M(\mathbf{v}) \exp \left(-\frac{\psi^{0}(t, \mathbf{x})}{\epsilon}-\psi^{1}(t, \mathbf{x}, \mathbf{v})-\epsilon \psi^{2}(t, \mathbf{x}, \mathbf{v})\right)}\right|=O\left(\epsilon^{2}\right)
$$

Therefore, we expect that the proposed method for approximating $f^{\epsilon}$ is $O\left(\epsilon^{2}\right)$ accurate. Numerical examples

in Section 4 verify the accuracy.

Remark 2. Assuming that $K$ Gauss abscissas/weights are used, and $O(N)$ mesh points are used in each spatial dimension (and in each velocity dimension for standard methods). At each time step, the complexity of the proposed method is $O\left(K^{n} N^{n}\right)$; while the complexity of standard methods is $O\left(N^{2 n}\right)$. In practice, we have $K \ll N$. And the proposed method does not depend on $\epsilon$. Compared to the standard methods, the proposed method has a much lower complexity without losing accuracy.

The proposed methodologies associated with the simpler BGK operator (1) is potentially extendable to more general BGK models, which will be presented and analyzed in a forthcoming paper.

\section{Numerical Examples}

Several numerical experiments are performed to demonstrate the accuracy of the proposed method. We record both the relative $l_{\infty}$ and $l_{1}$ errors between the solutions $f^{\epsilon}$ by the proposed method and the reference solutions $f^{\epsilon, r e f}$, i.e.,

$$
\frac{\left\|f^{\epsilon}(t, \mathbf{x}, \mathbf{v})-f^{\epsilon, r e f}(t, \mathbf{x}, \mathbf{v})\right\|_{\infty}}{\left\|f^{\epsilon, r e f}(t, \mathbf{x}, \mathbf{v})\right\|_{\infty}}, \frac{\left\|f^{\epsilon}(t, \mathbf{x}, \mathbf{v})-f^{\epsilon, r e f}(t, \mathbf{x}, \mathbf{v})\right\|_{1}}{\|f \epsilon, r e f(t, \mathbf{x}, \mathbf{v})\|_{1}}
$$

The convergence order (conv. order) is also recorded. The reference solutions are computed with the implicit-explicit Runge-Kutta (IMEX-RK) scheme in [21]. Numerical plots of the density function $f^{\epsilon}$, the macroscopic density $\rho^{\epsilon}$ and the momentum

$$
\mathbf{j}^{\epsilon}(t, \mathbf{x})=\int_{\mathbb{V}} \mathbf{v} f^{\epsilon}(t, \mathbf{x}, \mathbf{v}) d \mathbf{v}
$$


are presented for further comparisons.

For evaluating integrals in the velocity variable when applying Algorithms 1 and 2 , we use a Gauss quadrature rule with $M(\mathbf{v})$ as the weight function. The abscissas/weights can be computed with the algorithm in [10.

Example 1. We use 1-D examples to demonstrate the accuracy of the proposed method.

- 1-D case 1: $\Omega=[-1,1], \mathbb{V}=[-1,1], M(v) \equiv 0.5, \psi^{0}(0, x)=0.05(1+\sin (\pi(1+x))), \lambda(0, x)=$ $\cos (2 \pi(1+x))-1$, and $\beta(0, x)=1-\cos (2 \pi(1+x))$. Table 1 shows the comparisons between the computed solutions and the reference solutions.

- 1-D case 2: $\Omega=[-1,1], \mathbb{V}=[-2,2], M(v)=e^{-v^{2}} / \sqrt{\pi} \operatorname{erf}(2), \psi^{0}(0, x)=0.05(1+\cos (\pi(1+x)))$, $\lambda(0, x)=\sin (2 \pi(1+x))-1$, and $\beta(0, x)=1-\sin (2 \pi(1+x))$. Table 2 shows the comparisons between the computed solutions and the reference solutions. Figure 1 shows numerical plots of the solutions.

- 1-D case 3: $\Omega=[-1,1], \mathbb{V}=[-4,4], M(v)=e^{-v^{2}} / \sqrt{\pi} \operatorname{erf}(4), \psi^{0}(0, x)=0.05(1+\sin (\pi(1+x)))$, $\lambda(0, x)=\cos (2 \pi(1+x))-1$, and $\beta(0, x)=1-\sin (2 \pi(1+x))$. Table 3 shows the comparisons between the computed solutions and the reference solutions. Figure 2 shows numerical plots of the solutions.

For evaluating integrals in the velocity variable in Algorithms 1 and 2 , 16 Gauss abscissas/weights are used. Tables 1, 2 and 3 demonstrate that the proposed method is $O\left(\epsilon^{2}\right)$ accurate.

\begin{tabular}{|c|c|c|c|c|c|}
\hline$\epsilon=1 e-1$ & $\epsilon$ & $\epsilon / 2$ & $\epsilon / 4$ & $\epsilon / 8$ & $\epsilon / 16$ \\
\hline Error $\backslash$ mesh & 11 & 41 & 161 & 641 & 2561 \\
\hline \hline rel. $l_{\infty}$ & $4.69 e-1$ & $2.11 e-1$ & $6.33 e-2$ & $1.03 e-2$ & $1.62 e-3$ \\
\hline conv. order. & - & 1.15 & 1.74 & 2.62 & 2.67 \\
\hline rel. $l_{1}$ & $1.72 e-1$ & $8.26 e-2$ & $2.25 e-2$ & $5.05 e-3$ & $9.12 e-4$ \\
\hline conv. order. & - & 1.06 & 1.88 & 2.16 & 2.47 \\
\hline
\end{tabular}

Table 1: $1-\mathrm{D}$ example case 1 . The relative $l_{\infty}$ and $l_{1}$ errors are recorded at $t=0.5$.

\begin{tabular}{|c|c|c|c|c|c|}
\hline$\epsilon=1 e-1$ & $\epsilon / 2$ & $\epsilon / 4$ & $\epsilon / 8$ & $\epsilon / 16$ & $\epsilon / 32$ \\
\hline Error $\backslash$ mesh & 41 & 161 & 641 & 2561 & 10241 \\
\hline \hline rel. $l_{\infty}$ & $1.68 e-1$ & $5.25 e-2$ & $1.64 e-2$ & $4.79 e-3$ & $1.27 e-3$ \\
\hline conv. order. & - & 1.68 & 1.68 & 1.78 & 1.92 \\
\hline rel. $l_{1}$ & $1.46 e-1$ & $4.06 e-2$ & $1.11 e-2$ & $3.16 e-3$ & $9.11 e-4$ \\
\hline conv. order. & - & 1.85 & 1.87 & 1.81 & 1.79 \\
\hline
\end{tabular}

Table 2: 1-D example case 2. The relative $l_{\infty}$ and $l_{1}$ errors are recorded at $t=0.5$.

Example 2. We use 2-D examples to demonstrate the method.

- 2-D case 1: $\Omega=[-1,1] \times[-1,1], \mathbb{V}=[-2,2] \times[-2,2], M(u, v)=e^{-\left(u^{2}+v^{2}\right)} / \pi \operatorname{erf}^{2}(2), \psi^{0}(0, x, y)=$ $0.025(2-\sin (\pi(1+x))-\cos (\pi(1+y))), \lambda(0, x, y)=\cos (\pi(1+x))+\cos (\pi(1+y))-2$, and $\beta(0, x, y)=$ 


\begin{tabular}{|c|c|c|c|c|c|}
\hline$\epsilon=1 e-1$ & $\epsilon$ & $\epsilon / 2$ & $\epsilon / 4$ & $\epsilon / 8$ & $\epsilon / 16$ \\
\hline Error $\backslash$ mesh & 11 & 41 & 161 & 641 & 2561 \\
\hline \hline rel. $l_{\infty}$ & $5.24 e-1$ & $2.34 e-1$ & $8.47 e-2$ & $1.71 e-2$ & $1.47 e-3$ \\
\hline conv. order. & - & 1.16 & 1.47 & 2.31 & 3.54 \\
\hline rel. $l_{1}$ & $3.60 e-1$ & $1.66 e-1$ & $4.87 e-2$ & $1.09 e-2$ & $1.72 e-3$ \\
\hline conv. order. & - & 1.12 & 1.77 & 2.16 & 2.66 \\
\hline
\end{tabular}

Table 3: 1-D example case 3. The relative $l_{\infty}$ and $l_{1}$ errors are recorded at $t=0.5$.

a)

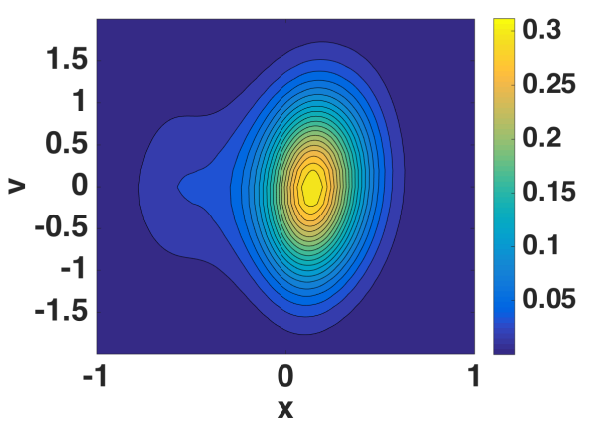

c)

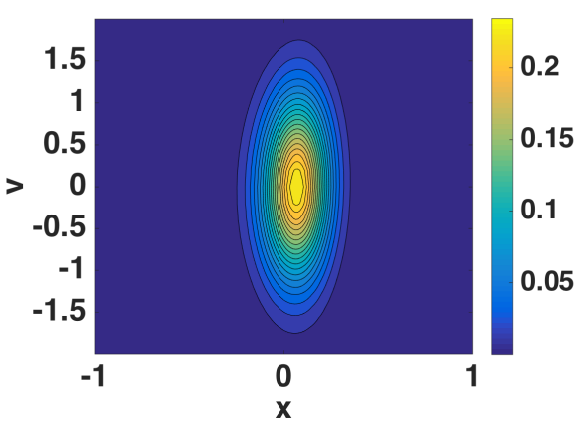

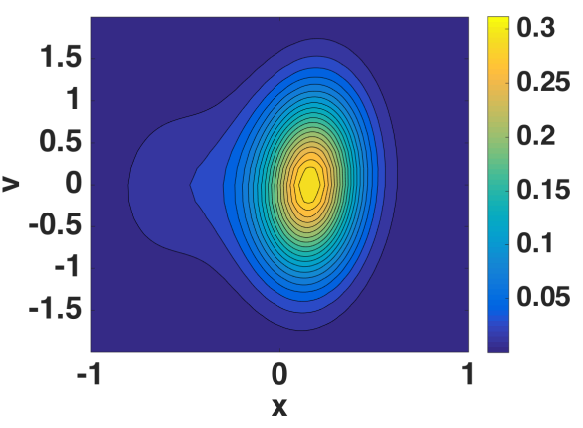

b)

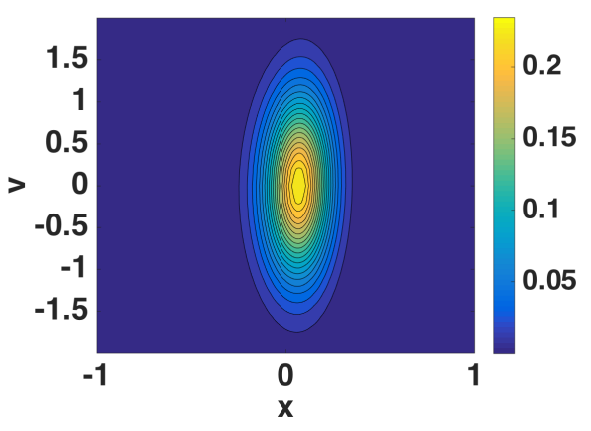

Figure 1: 1-D example case 2: solution at $t=0.5$ with $\epsilon=\frac{1}{40}$ (Top) and $\epsilon=\frac{1}{160}$ (Bottom). (a)-(c): plots of $f^{\epsilon}$ by proposed method; and (b)-(d): plots of $f^{\epsilon}$ by reference solution. Spatial mesh for the proposed method is 161 for both $\epsilon^{\prime}$; while spatial mesh for the reference solution is 161 for $\epsilon=\frac{1}{40}$ and 2561 for $\epsilon=\frac{1}{160}$. 


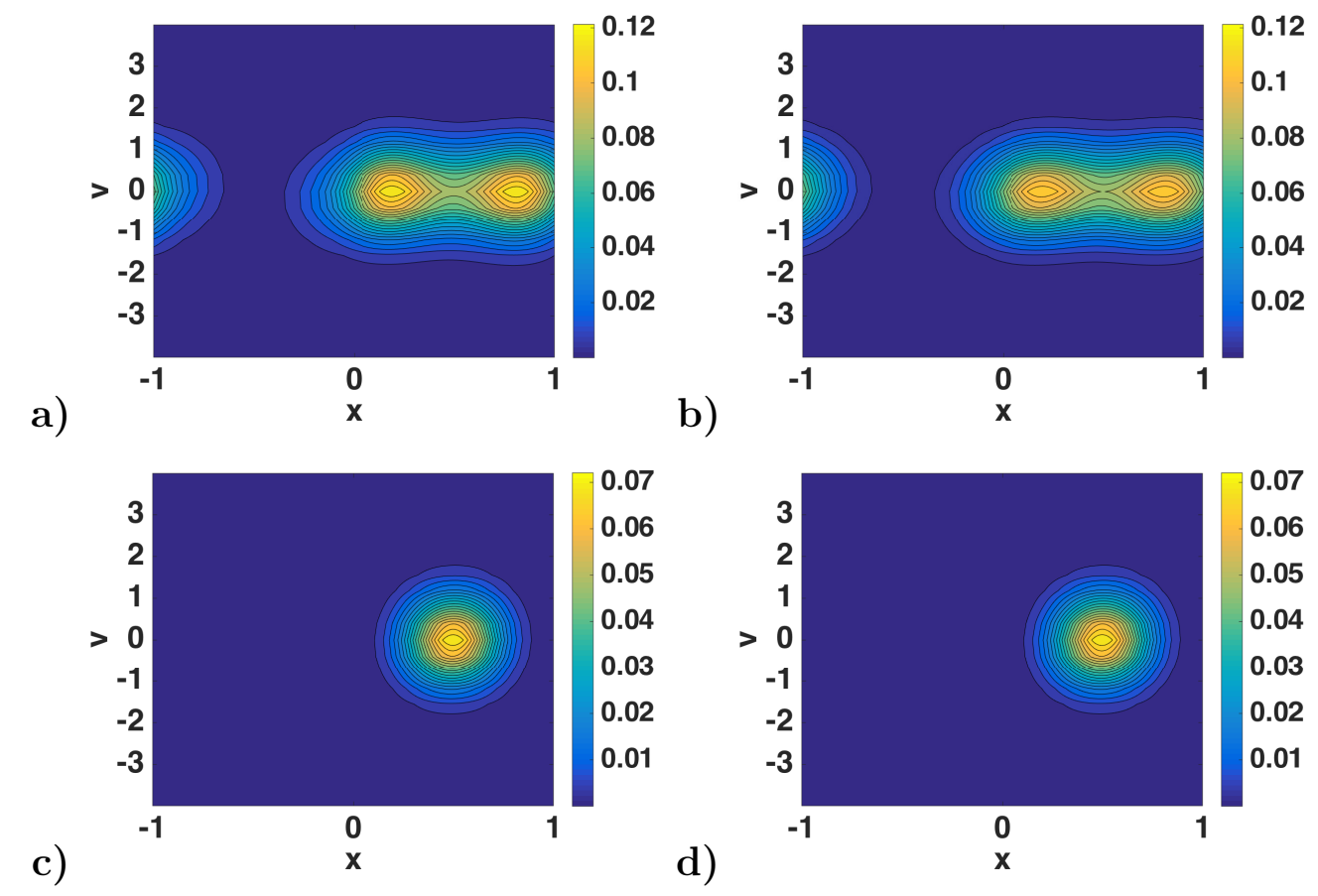

Figure 2: 1-D example case 3: solution at $t=0.5$ with $\epsilon=\frac{1}{40}$ (Top) and $\epsilon=\frac{1}{160}$ (Bottom). (a)-(c): plots of $f^{\epsilon}$ by proposed method; and (b)-(d): plots of $f^{\epsilon}$ by reference solution. Spatial mesh for the proposed method is 161 for both $\epsilon$ 's; while spatial mesh for the reference solution is 161 for $\epsilon=\frac{1}{40}$ and 2561 for $\epsilon=\frac{1}{160}$. 
$2-\cos (\pi(1+x))+\cos (\pi(1+y))$. Table 4 shows the comparisons between the computed solutions and the reference solutions. Figure 3 shows the plots of the numerical solutions.

- 2-D case 2: $\Omega=[-1,1] \times[-1,1], \mathbb{V}=[-4,4] \times[-4,4], M(u, v)=e^{-\left(u^{2}+v^{2}\right)} / \pi \operatorname{erf}^{2}(4), \psi^{0}(0, x, y)=$ $0.025(2-\cos (\pi(1+x))-\cos (\pi(1+y))), \lambda(0, x, y)=\sin (\pi(1+x))+\cos (\pi(1+y))-2$, and $\beta(0, x, y)=$ $2-\cos (\pi(1+x))-\sin (\pi(1+y))$. Table 5 shows the comparisons between the computed solutions and the reference solutions. Figure 4 shows the plots of the numerical solutions.

For evaluating integrals in the velocity variable in Algorithms 1 and 2 16 Gauss abscissas/weights are used. Tables 4 and 5 demonstrate that the proposed method is $O\left(\epsilon^{2}\right)$ accurate.

\begin{tabular}{|c|c|c|c|c|c|}
\hline$\epsilon=1 e-1$ & $\epsilon$ & $\epsilon / 2$ & $\epsilon / 4$ & $\epsilon / 8$ & $\epsilon / 16$ \\
\hline Error $\backslash$ mesh & $11 \times 11$ & $21 \times 21$ & $41 \times 41$ & $81 \times 81$ & $161 \times 161$ \\
\hline \hline rel. $l_{\infty}$ & $4.46 e-2$ & $1.24 e-2$ & $3.48 e-3$ & $7.89 e-4$ & $1.43 e-4$ \\
\hline conv. order. & - & 1.85 & 1.83 & 2.14 & 2.46 \\
\hline rel. $l_{1}$ & $4.79 e-2$ & $1.40 e-2$ & $3.63 e-3$ & $8.81 e-4$ & $2.25 e-4$ \\
\hline conv. order. & - & 1.77 & 1.95 & 2.04 & 1.97 \\
\hline
\end{tabular}

Table 4: $2-\mathrm{D}$ example case 1 . The relative $l_{\infty}$ and $l_{1}$ errors are recorded at $t=0.1$.

\begin{tabular}{|c|c|c|c|c|c|}
\hline$\epsilon=1 e-1$ & $\epsilon$ & $\epsilon / 2$ & $\epsilon / 4$ & $\epsilon / 8$ & $\epsilon / 16$ \\
\hline Error $\backslash$ mesh & $11 \times 11$ & $21 \times 21$ & $41 \times 41$ & $81 \times 81$ & $161 \times 161$ \\
\hline \hline rel. $l_{\infty}$ & $4.88 e-2$ & $1.37 e-2$ & $3.84 e-3$ & $8.54 e-4$ & $1.54 e-4$ \\
\hline conv. order. & - & 1.83 & 1.83 & 2.17 & 2.47 \\
\hline rel. $l_{1}$ & $4.21 e-2$ & $1.23 e-2$ & $3.18 e-3$ & $7.87 e-4$ & $2.03 e-4$ \\
\hline conv. order. & - & 1.76 & 1.95 & 2.01 & 1.95 \\
\hline
\end{tabular}

Table 5: 2-D example case 2 . The relative $l_{\infty}$ and $l_{1}$ errors are recorded at $t=0.1$.

Example 3. We present a 3-D example with $\Omega=\left[\begin{array}{ll}-1,1 & 3\end{array}\right]^{3} \mathbb{V}=[-2,2]^{3}, \psi^{0}(0, \mathbf{x})=0.025(3-$ $\sin (\pi(1+x))-\cos (\pi(1+y))-\sin (\pi(1+z))), \lambda(0, \mathbf{x})=-(3-\sin (\pi(1+x))-\sin (\pi(1+y))-\sin (\pi(1+z)))$, $\beta(0, \mathbf{x})=3-\cos (\pi(1+x))-\cos (\pi(1+y))-\cos (\pi(1+z))$, and $M(\mathbf{v})=e^{-\mathbf{v}^{2}} / \pi^{3 / 2} \operatorname{erf}^{3}(2)$. For evaluating integrals in the velocity variable in Algorithms 1 and 2, 8 Gauss abscissas/weights are used. Figure 5 shows the plots of the numerical solutions.

\section{Conclusions}

We present an asymptotic approach to numerically solving kinetic equations with a simple BGK relaxation operator in the large scale hyperbolic limit. Through an Hopf-Cole transformation, the computation of the density of particles is switched to the approximation of the phase function. The phase function is expanded as a power series with respect to the Knudsen number. The leading order term is the viscosity solution 
a)

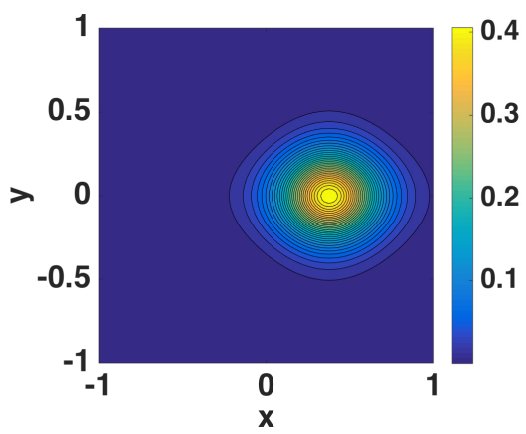

c)

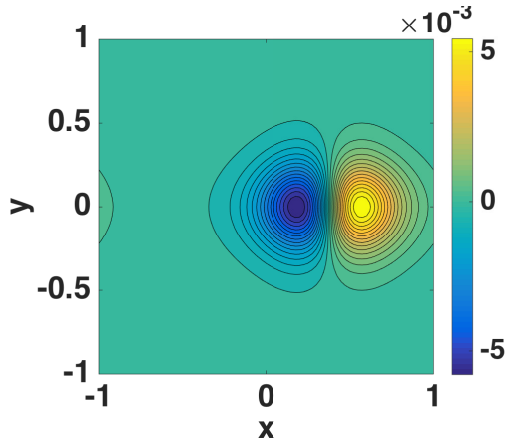

e)

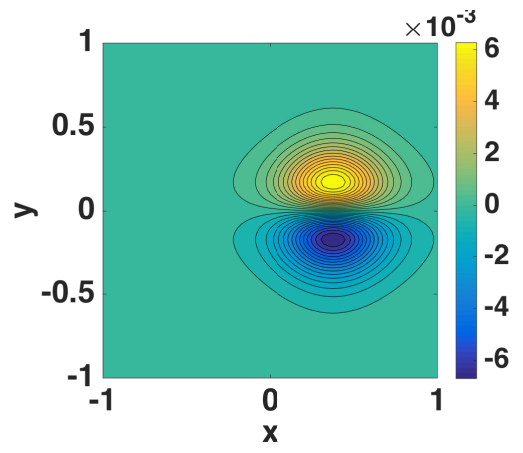

b)

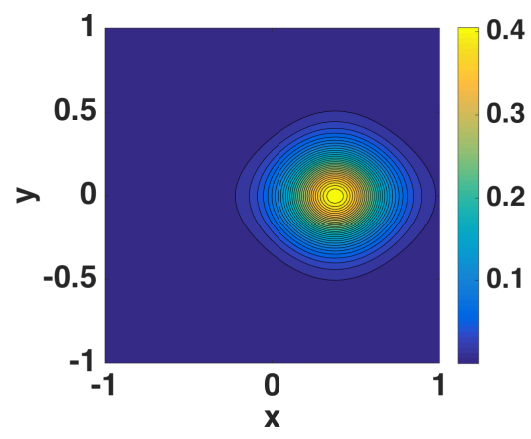

d)

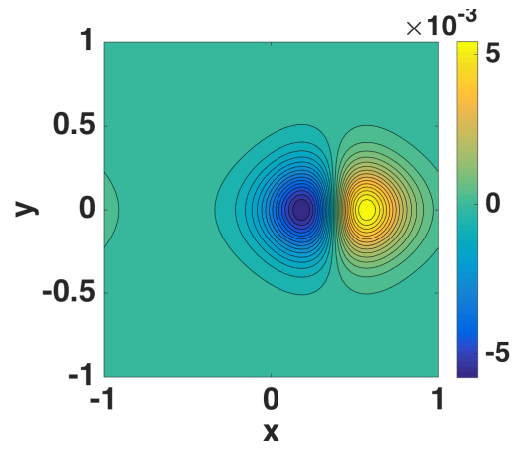

f)

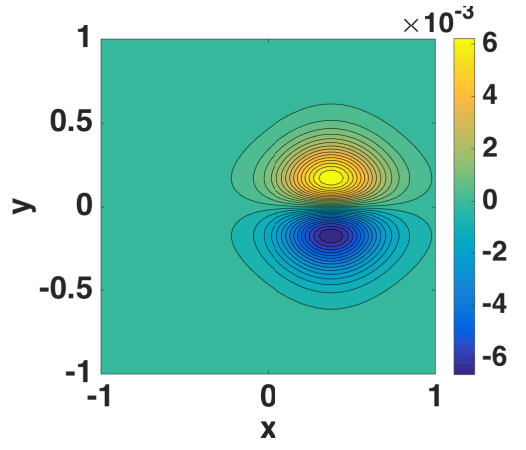

Figure 3: 2-D example case 1: solution at $t=0.2$ with $\epsilon=0.01$. (a)-(b): plots of $\rho^{\epsilon}$ by proposed method and reference solution respectively; (c)-(d): plots of $\mathbf{j}^{\epsilon}$ (first component) by proposed method and reference solution respectively; and (e)-(f): plots of $\mathbf{j}^{\epsilon}$ (second component) by proposed method and reference solution respectively. Spatial mesh: $81 \times 81$. 
a)

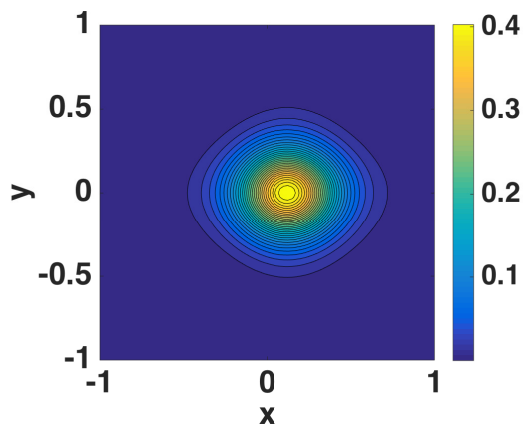

c)

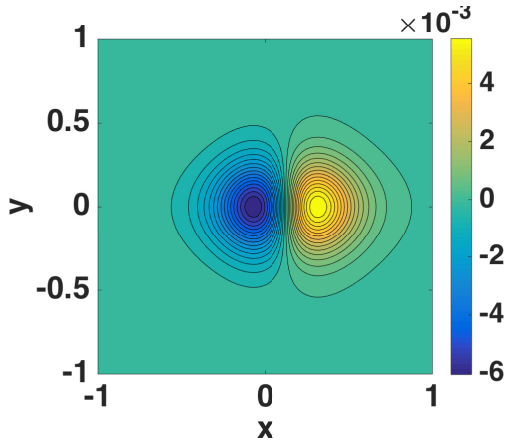

e)

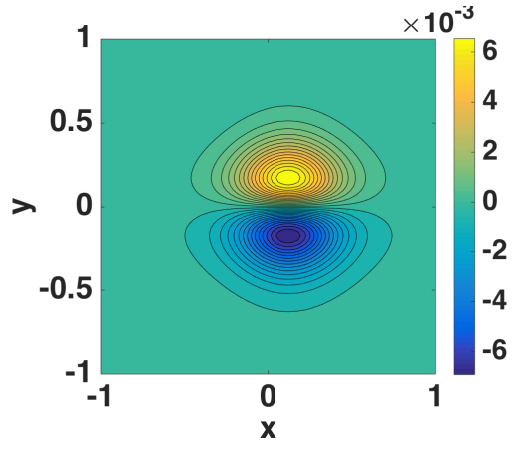

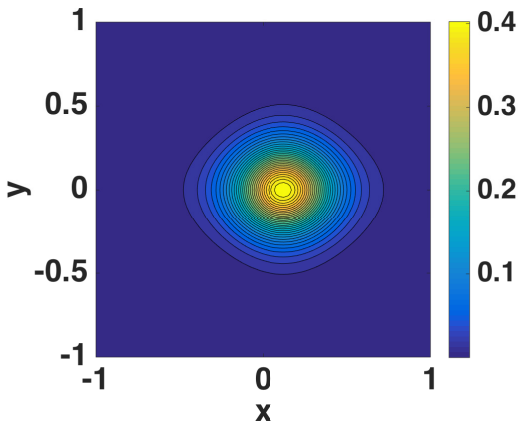

b)

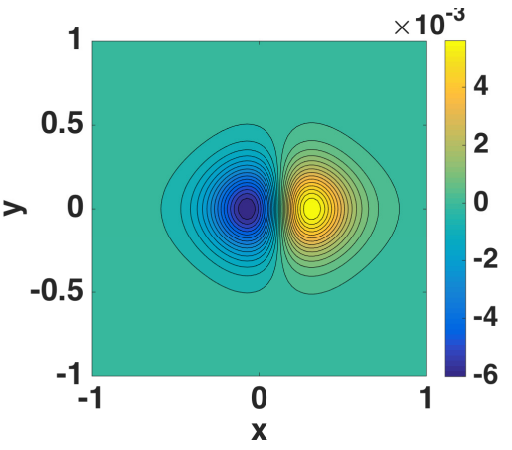

d)

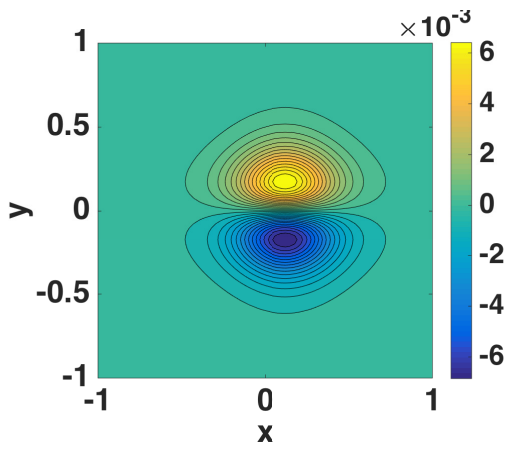

Figure 4: 2-D example case 2: solution at $t=0.2$ with $\epsilon=0.01$. (a)-(b): plots of $\rho^{\epsilon}$ by proposed method and reference solution respectively; (c)-(d): plots of $\mathbf{j}^{\epsilon}$ (first component) by proposed method and reference solution respectively; and (e)-(f): plots of $\mathbf{j}^{\epsilon}$ (second component) by proposed method and reference solution respectively. Spatial mesh: $81 \times 81$. 


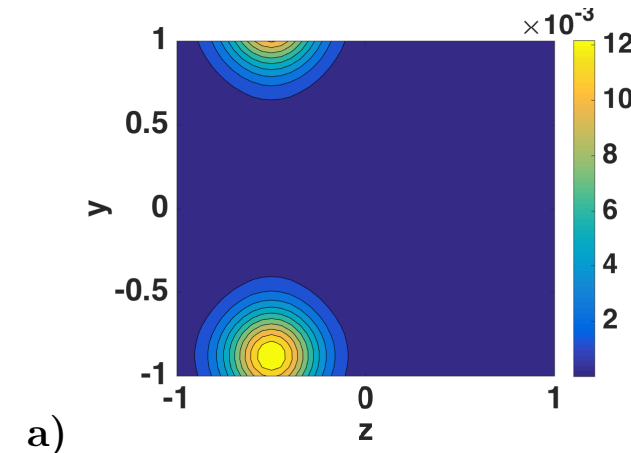

a)

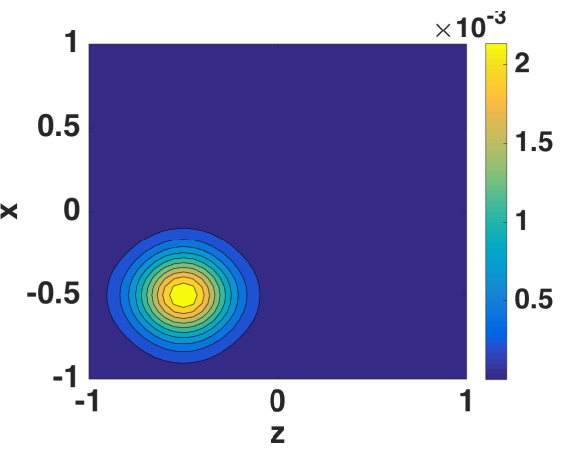

c)

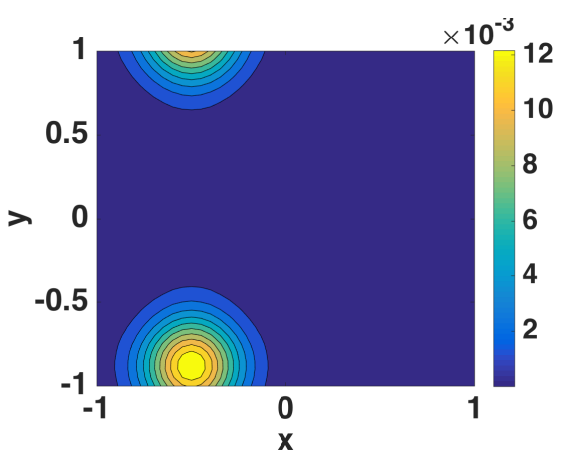

b)

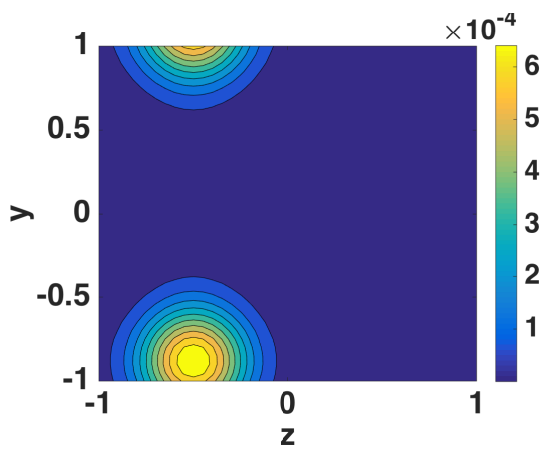

d)
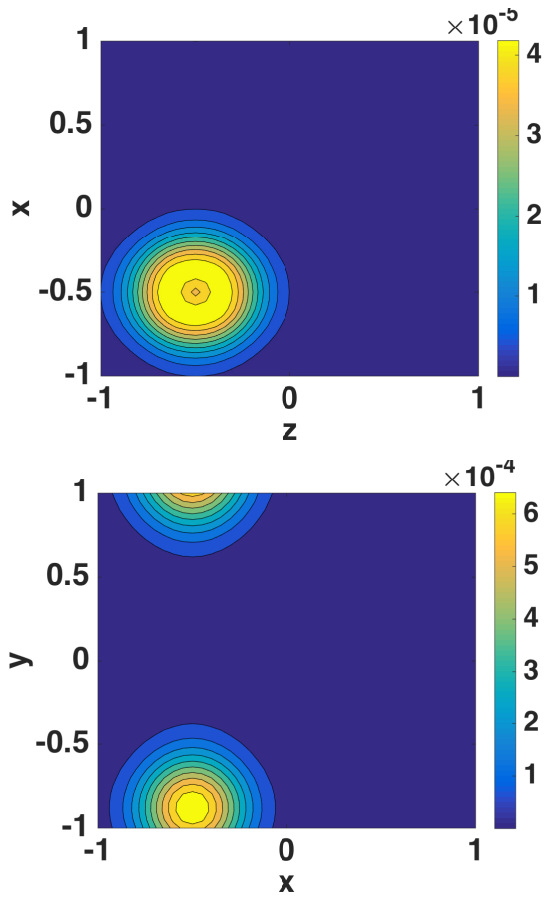

Figure 5: 3-D example: solution at $t=0.1$ with $\epsilon=0.01$. (a)-(c)-(e): plots of $\rho^{\epsilon}$ by proposed method at $x=0, y=0$ and $z=0$ respectively; and (b)-(d)-(f): plots of $\left\|\mathbf{j}^{\epsilon}\right\|_{2}$ by proposed method at $x=0, y=0$ and $z=0$ respectively. 
of an effective Hamilton-Jacobi equation, and the high order terms can be determined with solutions of a sequence of transport equations. These equations can be solved efficiently in the physical space since they are independent of the Knudsen number and their coefficients are given as integrals in the velocity variable that can be evaluated efficiently by Gauss Quadrature rules. In this work, zeroth, first and second order terms in the expansion are used and examined. Numerical experiments verify the efficiency of the method and demonstrate that the method has expected second order accuracy with respect to the Knudsen number.

The proposed asymptotic approach shows potentials to deal with kinetic equations with more general BGK operators, which is more desirable for real applications. Several projects will be pursued in the future: (1) higher order terms in the power series expansion will be included in the approximation to obtain higher order accuracy; and (2) the asymptotic approach will be extended to solve kinetic equations with more general BGK operators. For instance, we will consider the classical BGK model

$$
\partial_{t} f^{\epsilon}(t, \mathbf{x}, \mathbf{v})+\mathbf{v} \cdot f^{\epsilon}(t, \mathbf{x}, \mathbf{v})=\frac{1}{\epsilon}\left(f_{M}^{\epsilon}(t, \mathbf{x}, \mathbf{v})-f^{\epsilon}(t, \mathbf{x}, \mathbf{v})\right), t \geq 0
$$

with

$$
f_{M}^{\epsilon}(t, \mathbf{x}, \mathbf{v})=\rho^{\epsilon}(t, \mathbf{x}) M(t, \mathbf{x}, \mathbf{v}), M(t, \mathbf{x}, \mathbf{v})=\frac{1}{(2 \pi R T(t, \mathbf{x}))^{n / 2}} \exp \left(-\frac{\|\mathbf{v}-\mathbf{u}(t, \mathbf{x})\|_{2}^{2}}{2 R T(t, \mathbf{x})}\right),
$$

where $\rho^{\epsilon}, \mathbf{u}$ and $T$ are, respectively, the macroscopic density, velocity, and temperature of the gas, and they are obtained from the moments of $f^{\epsilon}$ that are defined as

$$
\left(\begin{array}{c}
\rho^{\epsilon}(t, \mathbf{x}) \\
\mathbf{m}(t, \mathbf{x}) \\
E(t, \mathbf{x})
\end{array}\right)=\int_{\mathbb{V}} f^{\epsilon}(t, \mathbf{x}, \mathbf{v})\left(\begin{array}{c}
1 \\
\mathbf{v} \\
\frac{1}{2}\|\mathbf{v}\|^{2}
\end{array}\right) d \mathbf{v} .
$$

Here $\mathbf{m}$ is the momentum, and $E$ is the total energy. The macroscopic velocity is $\mathbf{u}=\mathbf{m} / \rho^{\epsilon}$, and through internal energy $e$ one can obtain the temperature with $\rho^{\epsilon} e=E-\rho^{\epsilon} \mathbf{u}^{2} / 2, e=n R T / 2$. Similar equations in the physical space for determining the terms in the power series expansion will be derived and implemented.

\section{Acknowledgement}

SL would like to thank Dr. Yifeng Yu for fruitful discussion that improves this work.

\section{References}

[1] M. Bardi and S. J. Osher, The nonconvex multi-dimensional Riemann problem for Hamilton-Jacobi equations, SIAM J. Math. Analy. 22 (1991), 344-351. 
[2] P. L. Bhatnagar, E. P. Gross, and M. Krook, A Model for Collision Processes in Gases. I. Small Amplitude Processes in Charged and Neutral One-Component Systems, Phys. Rev. 94 (1954), 511-525.

[3] E. Bouin and V. Calvez, A kinetic eikonal equation, C. R. Acad. Sci. Paris, Ser. I 350 (2012), 243-248.

[4] N. Caillerie, Large deviations for a velocity jump process with a Hamilton-Jacobi approach, Comptes Rendus Mathematique 355 (2017), no. 2, 170-175.

[5] S. Chapman and T. G. Cowling, The Mathematical Theory of Non-uniform Gases: An Account of the Kinetic Theory of Viscosity, Thermal Conduction and Diffusion in Gases, Cambridge University Press, 1990.

[6] F. Coron and B. Perthame, Numerical Passage from Kinetic to Fluid Equations, SIAM J. Numer. Anal. 28 (1991), no. 1, 26-42.

[7] M. G. Crandall and P.-L. Lions, Two approximations of solutions of Hamilton-Jacobi equations, Math. Comp. 43 (1984), 1-19.

[8] G. Dimarco and L. Pareschi, Numerical methods for kinetic equations, Acta Numerica 23 (2014), 369520.

[9] B. Engquist and O. Runborg, Computational high frequency wave propagation, Acta Numerica 12 (2003), $181-266$.

[10] G. H. Golub and J. H. Welsch, Calculation of gauss quadrature rules, Math. Comp. 23 (1969), no. 106, $221-\mathrm{s} 10$.

[11] E. Hopf, The partial differential equation $u_{t}+u u_{x}=\mu_{x x}$, Communications on Pure and Applied Mathematics 3 (1950), no. 3, 201-230.

[12] G. S. Jiang and C. W. Shu, Efficient implementation of weighted ENO schemes, J. Comput. Phys. 126 (1996), 202-228.

[13] J. Keller and R. Lewis, Asymptotic methods for partial differential equations: the reduced wave equation and Maxwell's equations, Surv. in Appl. Math. 1 (1995), 1-82.

[14] J. B. Keller, Semiclassical mechanics, SIAM Review 27 (1985), 485-504.

[15] J. D. Lawson, An Order Five Runge Kutta Process with Extended Region of Stability, SIAM J. Numer. Anal. 3 (1966), 593-597. 
[16] X. D. Liu, S. J. Osher, and T. Chan, Weighted Essentially NonOscillatory schemes, J. Comput. Phys. 115 (1994), 200-212.

[17] S. Luo and N. Payne, Properties-preserving high order numerical methods for a kinetic eikonal equation, Journal of Computational Physics 331 (2017), 73-89.

[18] S. Luo and J. Qian, Factored singularities and high-order Lax-Friedrichs sweeping schemes for pointsource traveltimes and amplitudes, J. Comput. Phys. 230 (2011), 4742-4755.

[19] S. Luo, J. Qian, and R. Burridge, Fast Huygens sweeping methods for Helmholtz equations in inhomogeneous media in the high frequency regime, J. Comput. Phys. 270 (2014), 378-401.

[20] S. Osher and C.-W. Shu, High-order essentially nonoscillatory schemes for Hamilton-Jacobi equations, SIAM J. Numer. Anal. 28 (1991), no. 4, 907-922.

[21] S. Pieraccini and G. Puppo, Implicit-Explicit Schemes for BGK Kinetic Equations, J. Sci. Comput. 32 (2007), no. 1, 1-28.

[22] J. Qian and W. W. Symes, Adaptive finite difference method for traveltime and amplitude, Geophysics 67 (2002), 167-176.

[23] C. W. Shu, Essentially non-oscillatory and weighted essentially non-oscillatory schemes for hyperbolic conservation laws, Advanced Numerical Approximation of Nonlinear Hyperbolic Equations (B. Cockburn, C. Johnson, C.W. Shu, and E. Tadmor, eds.), vol. 1697, Springer, 1998, Lecture Notes in Mathematics, pp. 325-432. 